\title{
HST/COS observations of the newly discovered obscuring outflow in NGC 3783
}

\author{
G. A. Kriss ${ }^{1}$, M. Mehdipour ${ }^{2}$, J. S. Kaastra ${ }^{2,3}$, A. Rau ${ }^{4}$, J. Bodensteiner ${ }^{4,5}$, R. Plesha ${ }^{1}$, N. Arav ${ }^{6}$, \\ E. Behar ${ }^{7}$, S. Bianchi ${ }^{8}$, G. Branduardi-Raymont ${ }^{9}$, M. Cappi ${ }^{10}$, E. Costantini ${ }^{2}$, B. De Marco ${ }^{11}$, \\ L. Di Gesu ${ }^{12}$, J. Ebrero' ${ }^{13}$, S. Kaspi ${ }^{7}$, J. Mao ${ }^{2,3}$, R. Middei ${ }^{8}$, T. Miller ${ }^{6}$, S. Paltani ${ }^{12}$, U. Peretz ${ }^{7}$, \\ B. M. Peterson ${ }^{1,14,15}$, P.-O. Petrucci ${ }^{16}$, G. Ponti ${ }^{17}$, F. Ursini ${ }^{10}$, D. J. Walton ${ }^{18}$, and X. $\mathrm{Xu}^{6}$ \\ 1 Space Telescope Science Institute, 3700 San Martin Drive, Baltimore, MD 21218, USA \\ e-mail: gak@stsci.edu \\ 2 SRON Netherlands Institute for Space Research, Sorbonnelaan 2, 3584 CA Utrecht, The Netherlands \\ 3 Leiden Observatory, Leiden University, PO Box 9513, 2300 RA Leiden, The Netherlands \\ 4 Max-Planck-Institut für Extraterrestriche Physik, Gießenbachstraße, 85748 Garching, Germany \\ 5 Institute of Astronomy, KU Leuven, Celestijnenlaan 200D bus 2401, 3001 Leuven, Belgium \\ ${ }^{6}$ Department of Physics, Virginia Tech, Blacksburg, VA 24061, USA \\ 7 Department of Physics, Technion-Israel Institute of Technology, 32000 Haifa, Israel \\ 8 Dipartimento di Matematica e Fisica, Università degli Studi Roma Tre, via della Vasca Navale 84, 00146 Roma, Italy \\ 9 Mullard Space Science Laboratory, University College London, Holmbury St. Mary, Dorking, Surrey RH5 6NT, UK \\ 10 INAF-IASF Bologna, Via Gobetti 101, 40129 Bologna, Italy \\ 11 Nicolaus Copernicus Astronomical Center, Polish Academy of Sciences, Bartycka 18, 00-716 Warsaw, Poland \\ 12 Department of Astronomy, University of Geneva, 16 Ch. d'Ecogia, 1290 Versoix, Switzerland \\ 13 European Space Astronomy Centre, PO Box 78, 28691 Villanueva de la Cañada, Madrid, Spain \\ 14 Department of Astronomy, The Ohio State University, 140 West 18th Ave., Columbus, OH 43210, USA \\ 15 Center for Cosmology \& AstroParticle Physics, The Ohio State University, 191 West Woodruff Ave., Columbus, OH 43210, USA \\ 16 Univ. Grenoble Alpes, CNRES, IPAG, 38000 Grenoble, France \\ 17 INAF-Osservatorio Astronomico di Brera, Via E. Bianchi 46, 23807 Merate, LC, Italy \\ 18 Institute of Astronomy, Madingley Road, CB3 OHA Cambridge, UK
}

Received 26 September 2018 / Accepted 29 October 2018

\begin{abstract}
Aims. To understand the nature of transient obscuring outflows in active galactic nuclei, we use simultaneous multiwavelength observations with XMM-Newton, NuSTAR, the Hubble Space Telescope (HST), and the Max Planck Gesellschaft/European Southern Observatory (ESO) $2.2 \mathrm{~m}$ telescope triggered by soft X-ray absorption detected by Swift.

Methods. We obtained ultraviolet spectra on 2016-12-12 and 2016-12-21 using the Cosmic Origins Spectrograph (COS) on HST simultaneously with X-ray spectra obtained with XMM-Newton and NuSTAR. We modeled the ultraviolet spectra to measure the strength and variability of the absorption, and used photoionization models to obtain its physical characteristics.

Results. We find new components of broad, blue-shifted absorption associated with Ly $\alpha, \mathrm{N}$ v, Si iv, and C iv in our COS spectra. The absorption extends from near-zero velocities in the rest-frame of the host galaxy to $-6200 \mathrm{~km} \mathrm{~s}^{-1}$. These features appear for the first time in NGC 3783 at the same time as heavy soft X-ray absorption seen in the XMM-Newton X-ray spectra. The X-ray absorption has a column density of $\sim 10^{23} \mathrm{~cm}^{-2}$, and it partially covers the X-ray continuum source. Combining the X-ray column densities with the UV spectral observations yields an ionization parameter for the obscuring gas of $\log \xi=1.84_{-0.2}^{+0.4} \mathrm{erg} \mathrm{cm} \mathrm{s}^{-1}$. Despite the high intensity of the UV continuum in NGC 3783, $F(1470 \AA)=8 \times 10^{-14} \mathrm{erg} \mathrm{cm}^{-2} \mathrm{~s}^{-1} \AA^{-1}$, the well known narrow UV absorption lines are deeper than in earlier observations in unobscured states, and low ionization states such as C III appear, indicating that the narrow-line gas is more distant from the nucleus and is being shadowed by the gas producing the obscuration. Despite the high continuum flux levels in our observations of NGC 3783, moderate velocities in the UV broad line profiles have substantially diminished. Conclusions.We suggest that a collapse of the broad line region has led to the outburst and triggered the obscuring event.
\end{abstract}

Key words. ultraviolet: galaxies - galaxies: active - galaxies: Seyfert - galaxies: individual: NGC 3783 - quasars: absorption lines quasars: emission lines

\section{Introduction}

Outflows from active galactic nuclei (AGN) may be the regulating mechanism that links the growth of supermassive black holes at galaxy centers to the size of the host galaxy. A possible outcome of such a linkage is the correlation between central velocity dispersions in galaxies and the masses of their central black holes (Magorrian et al. 1998; Ferrarese \& Merritt
2000; Gebhardt et al. 2000; Kormendy \& Ho 2013). Feedback from outflows may also regulate the overall mass and size of the host galaxy (Silk \& Rees 1998; King 2003; Ostriker et al. 2010; Soker 2010; Faucher-Giguère \& Quataert 2012; Zubovas \& Nayakshin 2014; Thompson et al. 2015).

Outflows from AGN manifest themselves in a variety of forms, from narrowly collimated radio jets to broad, widespread winds. In the latter case, these winds are often identified 
via their broad, blue-shifted absorption features in X-ray and ultraviolet spectra (Crenshaw et al. 2003), or extended, red and blue-shifted emission-line regions (Liu et al. 2013a,b, 2014). Again, the mechanisms for these various manifestations may vary, from radiatively driven (Murray \& Chiang 1995, 1997; Proga et al. 2000; Thompson et al. 2015) or magnetically accelerated (Königl \& Kartje 1994; Fukumura et al. 2010) winds originating from the accretion disk, or thermal winds originating either from the accretion disk, the broad-line region, or the obscuring torus (Krolik \& Kriss 1995, 2001).

Understanding the physical properties of outflows to ascertain how they work is crucial to be able to model the interaction of central black holes with their host galaxies. Observationally, in the X-ray and the UV, outflows have appeared with a variety of characteristics, perhaps indicating several mechanisms may be at work. Examples include the X-ray warm absorbers and associated narrow UV absorption lines described by Crenshaw et al. (2003); ultrafast outflows, typically only visible as broad, highly blue-shifted Fe $\mathrm{K}$ features (Pounds et al. 2003; Reeves et al. 2009; Tombesi et al. 2010; Nardini et al. 2015); and the newly discovered obscuring outflows showing strong soft X-ray absorption accompanied by broad, fast, blueshifted UV absorption lines: NGC 5548 (Kaastra et al. 2014), Mrk 335 (Longinotti et al. 2013), NGC 985 (Ebrero et al. 2016), and most recently, NGC 3783 (Mehdipour et al. 2017).

In the case of obscuring outflows, the gas appears to be mildly ionized and has high column density $\left(10^{22}-10^{23} \mathrm{~cm}^{-2}\right)$. This produces strong soft X-ray absorption, but no visible spectral features that allow diagnostics of the kinematics or ionization state of the gas. The crucial element in all the cases cited above is the availability of contemporaneous UV spectra. The UV absorption lines that appear in these events provide the necessary diagnostics that show gas outflowing (blue-shifted) with velocities and ionization states consistent with an origin in, or interior to the broad-line region (BLR). With no UV spectra, such obscuration events would be indistinguishable from other X-ray eclipsing events as studied by Markowitz et al. (2014), which could be caused just as easily by clouds in transverse motion as opposed to having a significant outflow component.

To understand the nature of obscuring outflows better and study their potential relationship to X-ray eclipsing events, we undertook a monitoring program with Swift (Gehrels et al. 2004) to find potential obscuring events that we could then study in detail with multiwavelength observations using XMM-Newton (Jansen et al. 2001), NuSTAR (Harrison et al. 2013), and the Cosmic Origins Spectrograph (COS) on the Hubble Space Telescope (HST). Mehdipour et al. (2017) presented preliminary results from this campaign. They found that the obscuring gas has kinematics and physical characteristics comparable to gas normally associated with the BLR. Thus, further study of obscuring outflows may offer some insights into the physical structure of the BLR. Reverberation mapping (Blandford \& McKee 1982; Peterson 1993) of the BLR has indicated that motions are consistent with Keplerian motion in a gravitational field dominated by the central black hole (Krolik et al. 1991; Peterson \& Wandel 1999). Recent advances allowing two-dimensional reverberation mapping in both spatial and velocity dimensions (Horne et al. 2004; Bentz et al. 2010; Grier et al. 2013; Pancoast et al. 2014a,b) confirm the dominance of Keplerian motions, but also show evidence for inflows and outflows associated with the BLR. Models of accretion-disk winds have often suggested that the BLR may be an observational manifestation of such winds, either radiatively driven by line opacity (Murray \& Chiang 1995, 1997; Proga et al. 2000; Thompson et al. 2015) or by radiation pressure on dust (Czerny \& Hryniewicz 2011; Czerny et al. 2017; Baskin \& Laor 2018), or magnetohydrodynamic (Königl \& Kartje 1994; Fukumura et al. 2010). If obscuring outflows are related to winds such as those that produce the BLR, then their transient nature and its possible relationship to changes in the BLR may provide additional insights into how the BLR forms and evolves.

The Seyfert 1 galaxy NGC 3783 has been studied extensively in past UV and X-ray observational campaigns. The reverberation mapping campaign using the International Ultraviolet Explorer (IUE) and ground-based observatories in 1991-1992 (Reichert et al. 1994; Stirpe et al. 1994) established the size of the broad-line region (BLR) at 4-10lt-days based on the lags of prominent emission lines ( $\mathrm{Ly} \alpha, \mathrm{C}_{\mathrm{IV}}, \mathrm{Mg}$ II, $\mathrm{H} \beta$ ) relative to variations in the continuum emission. In 2000-2001 an intensive X-ray and UV monitoring campaign obtained many observations of NGC 3783 using Chandra (Kaspi et al. 2002), HST (Gabel et al. 2003a), and the Far Ultraviolet Spectroscopic Explorer (FUSE; Gabel et al. 2003a). The high-resolution X-ray spectra revealed details of the X-ray warm absorber (Kaspi et al. 2002; Netzer et al. 2003) and its relationship to the narrow intrinsic absorption lines (Gabel et al. 2003a,b, 2005). The intrinsic UV absorption lines comprised four discrete components at outflow velocities ranging from -1352 to $-539 \mathrm{~km} \mathrm{~s}^{-1}$, and the ensemble closely matched the kinematic appearance of the X-ray absorption lines in the Chandra spectra (Gabel et al. 2003a). Their absorption depth displays variations consistent with a photoionization response to changes in the UV continuum, and the density-sensitive $\mathrm{C}$ III* $\lambda 1176$ multiplet yielded a density of $\log n_{\mathrm{e}}=4.5 \mathrm{~cm}^{-3}$ for Component $\# 1\left(v=-1311 \mathrm{~km} \mathrm{~s}^{-1}\right)$, implying a distance of $25 \mathrm{pc}$ for the gas producing the narrow UV absorption lines (Gabel et al. 2005). In contrast with the narrow UV absorption lines common in other Seyfert galaxies (Crenshaw et al. 2003), Component \#1 has appeared to "decelerate", with its centroid evolving from an outflow velocity of $-1352 \mathrm{~km} \mathrm{~s}^{-1}$ to $-1043 \mathrm{~km} \mathrm{~s}^{-1}$ (Scott et al. 2014) over 14 years. This unusual kinematic behavior makes NGC 3783 an interesting object for continuing studies.

In this paper we describe the HST/COS observations and their analysis that were part of the detection of a new obscuring outflow in NGC 3783 by Mehdipour et al. (2017). In Sect. 2 we describe the UV and optical observations and our data reduction methods. In Sect. 3 we model the UV and optical spectra and present an analysis of the physical properties of the obscuring outflow and the evolution of the narrow intrinsic UV absorption lines over the past 15 years. In Sect. 4 we discuss the implications of our observations for the structure of the BLR and for the origin of the obscuring outflow and its potential influence on the host galaxy. In Sect. 5 we present our conclusions.

\section{Observations and data reduction}

During Swift Cycle 12 in 2016 November, we detected spectral hardening in NGC 3783 indicative of an obscuring event. We triggered coordinated XMM-Newton, NuSTAR, and HST/COS observations on 2016-12-12 (Visit 3 in Program 14481) and 2016-12-21 (Visit 4). In addition, on 2016-12-12 we obtained ground-based spectroscopy of the $\mathrm{H} \beta$ region using the Fiber-fed Extended Range Optical Spectrograph (FEROS) on the Max Planck Gesellschaft/European Southern Observatory (MPG/ESO) $2.2 \mathrm{~m}$ telescope. For a baseline spectral comparison to NGC 3783 in the unobscured state, we use archival HST UV 


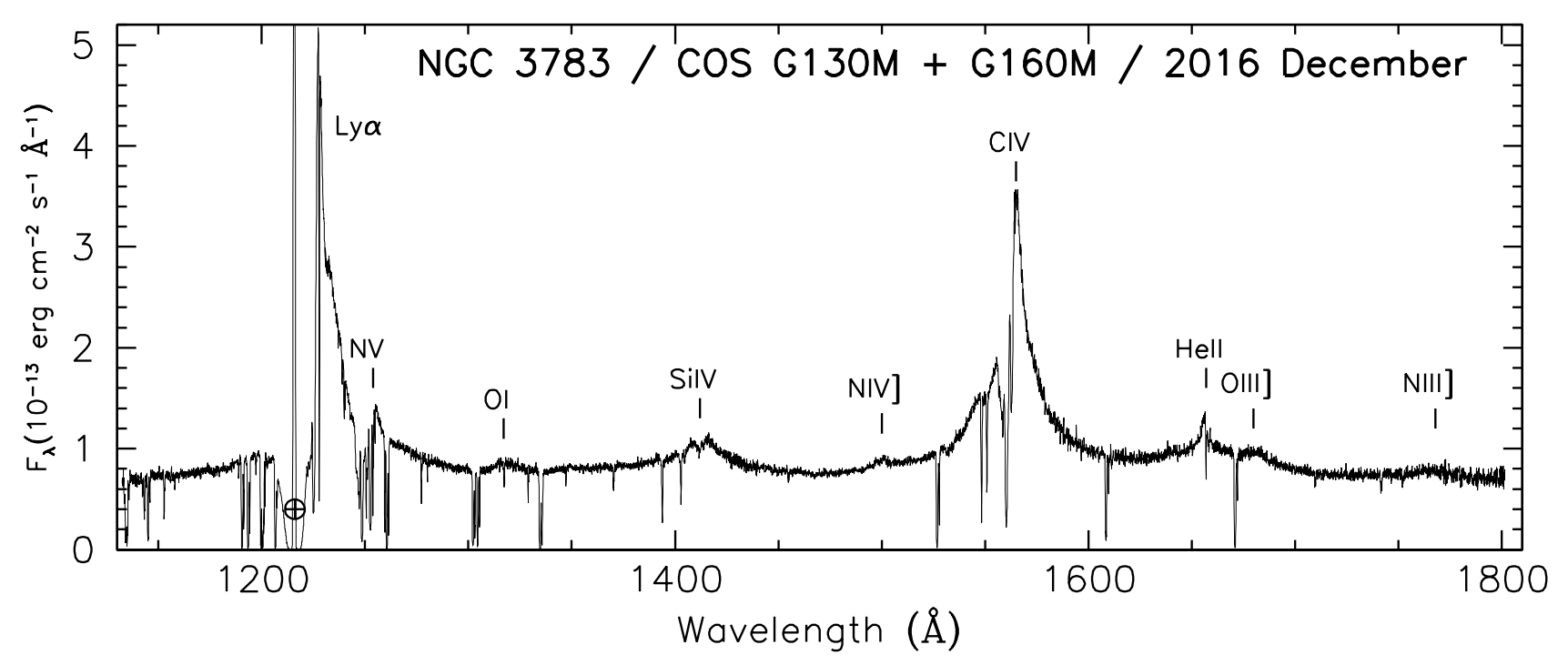

Fig. 1. Calibrated and merged COS spectrum of NGC 3783 from 2016 December. Data are binned by eight pixels, or approximately one resolution element. We label the most prominent emission features. Geocoronal emission in the center of the Milky Way Ly $\alpha$ absorption trough is indicated with an Earth symbol.

and optical spectra obtained with the Space Telescope Imaging Spectrograph (STIS; Woodgate et al. 1998).

\subsection{COS Observations}

Each COS observation consisted of a two-orbit visit using the gratings G130M and G160M to cover the 1130-1800 $\AA$ wavelength range at a resolving power of $\sim 15000$ (Green et al. 2012). We used multiple central wavelength settings and multiple FP-POS positions to cover the gaps between detectors A and $\mathrm{B}$ for each grating, and to sample the spectrum on different sections of each detector to allow for removal of detector artifacts and other flat-field anomalies. Table A.1 provides a summary of the various $\mathrm{COS}$ exposures.

Using updated wavelength calibrations, flat fields, and flux calibrations, we reprocessed our individual exposures as described by Kriss et al. (2011) and De Rosa et al. (2015). We then cross-correlated each exposure with prior HST/STIS observations of NGC 3783 (Gabel et al. 2003a) to adjust the zero-points of their wavelength scales before combining them into merged spectra representing each visit, as well as the two 2016 visits combined. Figure 1 shows the full merged spectrum from both 2016 visits. Since NGC 3783 was exceptionally bright during both of our visits (mean flux $F(1470 \AA)=$ $7.6 \times 10^{-14} \mathrm{erg} \mathrm{cm}^{-2} \mathrm{~s}^{-1} \AA^{-1}$ compared to the historical average of $5.0 \times 10^{-14} \mathrm{erg} \mathrm{cm}^{-2} \mathrm{~s}^{-1} \AA^{-1}$; Dunn et al. 2006), each merged spectrum achieves a signal-to-noise ratio $(\mathrm{S} / \mathrm{N})$ exceeding 40 per resolution element over the wavelength range from Ly $\alpha$ to the $\mathrm{C}$ Iv line.

\subsection{FEROS Observations}

We observed NGC 3783 with the Fiber-fed Extended Range Optical Spectrograph (Kaufer et al. 1999; FEROS) at the MPG/ESO $2.2 \mathrm{~m}$ telescope at the ESO La Silla Observatory. Our spectra covered the wavelength range 3500-9200 $\AA$ at a resolving power of 48000 . Starting at 07:36 GMT on 2016$12-12$, three $10 \mathrm{~min}$ exposures were performed successively. Wavelength calibration frames were obtained with ThArNe lamps.
The FEROS spectra were reduced manually using the standard pipeline (FEROS-DRS ${ }^{1}$ ) based on ESO-MIDAS. Before the actual data reduction, the three exposures were averaged and a cosmic ray rejection was performed. The successive data reduction included flat fielding, bias and background subtraction, and wavelength calibration.

\subsection{Archival HST Data}

In 2000-2001 an intensive X-ray and UV monitoring campaign obtained many observations of NGC 3783 using Chandra (Kaspi et al. 2002), HST (Gabel et al. 2003a), and the FUSE (Gabel et al. 2003a). We obtained the calibrated STIS spectra from the Mikulski Archive for Space Telescopes (MAST) and combined them as an unweighted average to use as our baseline for comparison of the new COS spectra.

In 2011, the HST program 12212 (PI: M. Crenshaw) obtained an optical long-slit spectrum of the nuclear region of NGC 3783 using the STIS CCD, grating G430M, and the $52 \times 0.2^{\prime \prime}$ slit. This spectrum covers the wavelength region surrounding $\mathrm{H} \beta$ and [O $\mathrm{III}]$, and we use it as our baseline for comparing our ground-based spectrum in the obscured state to an unobscured state. Table A.1 gives the observational details for these spectra. The STIS spectra were taken at three dithered slit positions to facilitate removal of cosmic rays and defective pixels. After correcting the data for charge-transfer inefficiency using a pixel-based algorithm based on Anderson \& Bedin (2010), we shifted the calibrated two-dimensional images to a common geometric position using integer-pixel shifts and combined them using a median filter to reject bad pixels. After this process, some cosmic ray residuals were still present. We identified these interactively, and interpolated across them using the fluxes in the adjacent pixels.

\section{Data analysis}

The absorption in the blue wings of the Ly $\alpha, \mathrm{Nv}$, Si Iv, and $\mathrm{C}_{\mathrm{IV}}$ emission lines in NGC 3783 is difficult to discern

\footnotetext{
1 https://www.eso.org/sci/facilities/lasilla/ instruments/feros/tools/DRS.html
} 
on the scale of Fig. 1, but it is more readily apparent in Fig. 3 of Mehdipour et al. (2017), where one can compare it directly to the high-quality STIS spectrum obtained in 2001 (Gabel et al. 2003a) during an unobscured epoch. To quantitatively assess the properties of the obscuration that appears in the COS spectrum, we start with a model of the emission lines and continuum based on the 2001 STIS spectrum. Our model is similar to the comprehensive model used for NGC 5548 (Kaastra et al. 2014) and consists of a power law continuum, $F_{\lambda}=F_{1000 \AA}(\lambda / 1000 \AA)^{-\alpha}$, that is reddened by fixed extinction of $E(B-V)=0.107$ (Schlafly \& Finkbeiner 2011), and Gaussian emission components (hereafter referred to as Gaussians) for each significant emission line. As usual, the bright components require several Gaussians. Ly $\alpha$ and C Iv are each comprised of 4 Gaussians: a narrow component with full-width at half-maximum $\left(F W H M \sim 900 \mathrm{~km} \mathrm{~s}^{-1}\right)$, a mediumbroad component $\left(F W H M \sim 2500 \mathrm{~km} \mathrm{~s}^{-1}\right)$, a broad component $\left(F W H M \sim 4500 \mathrm{~km} \mathrm{~s}^{-1}\right)$, and a very broad component $\left(F W H M \sim 10000 \mathrm{~km} \mathrm{~s}^{-1}\right) . \mathrm{N} \mathrm{v}, \mathrm{Si}$ IV and He II require only three components (narrow, medium broad or broad, and very broad), and the weaker lines usually require only a broad component, or a narrow plus a medium-broad to broad. For $\mathrm{N} v, \mathrm{Si}$ Iv, and $\mathrm{C}$ Iv, we allow for both lines in the doublets for the narrow, mediumbroad, and broad components, but only assume one component for the very broad line. We also assume the doublets are optically thick and have flux ratios of $1: 1$. The whole emission model is then absorbed by foreground Galactic $\mathrm{H}_{\mathrm{I}} \mathrm{Ly} \alpha$ as a damped Lorentzian profile with column density $\mathrm{N}(\mathrm{HI})=9.59 \times 10^{20} \mathrm{~cm}^{-2}$ (Murphy et al. 1996).

We note that this Gaussian decomposition is a semiempirical model and that the individual components do not necessarily represent physically distinct portions of the line-emitting regions. They also are neither independent nor orthogonal. However, in both NGC 5548 and NGC 3783, there are distinct narrow portions of the line profile that are stable over time in both width, velocity, and flux (Crenshaw et al. 2009). As we show later, the narrow-line components in the spectra at all epochs vary by $\lesssim 10 \%$. The lack of variability implies that these narrow components are likely representative of emission from the narrow-line region at some distance $(\sim 1 \mathrm{pc})$ from the nucleus. This is important since these narrow components are probably not absorbed by the obscurer.

We optimize parameters for each component in the model using the spectral fitting program specfit (Kriss 1994) in IRAF. The wavelength regions for our fits exclude all absorption lines, both intrinsic to NGC 3783, and the foreground lines from the interstellar medium (ISM). Since the intrinsic absorbers affect significant portions of the blue sides of all the lines, bestfit parameters are largely (but not exclusively) determined by the red sides of the profiles and the line wings $\left(>1500 \mathrm{~km} \mathrm{~s}^{-1}\right.$ from line center for the STIS spectrum, and $>6500 \mathrm{~km} \mathrm{~s}^{-1}$ for the 2016 COS spectra). For each spectrum, we optimized our fit in stages. The continuum normalization and power-law index were initially fixed using continuum regions near the blue and red ends of the spectrum. We initially fixed the line centers at the host galaxy systemic velocity, $z=0.00973$, a value determined via H I $21 \mathrm{~cm}$ measurements (Theureau et al. 1998). We interactively chose widths and fluxes for the lines that fit well by eye. Next, we let all the emission line fluxes vary. Once these have converged, we let the continuum parameters also vary freely to optimize its shape simultaneously with the broad wings of the emission lines. At this point the fit bore a good resemblance to the actual spectrum, but was not a good match in all details. To approach full convergence, we then successively addressed the

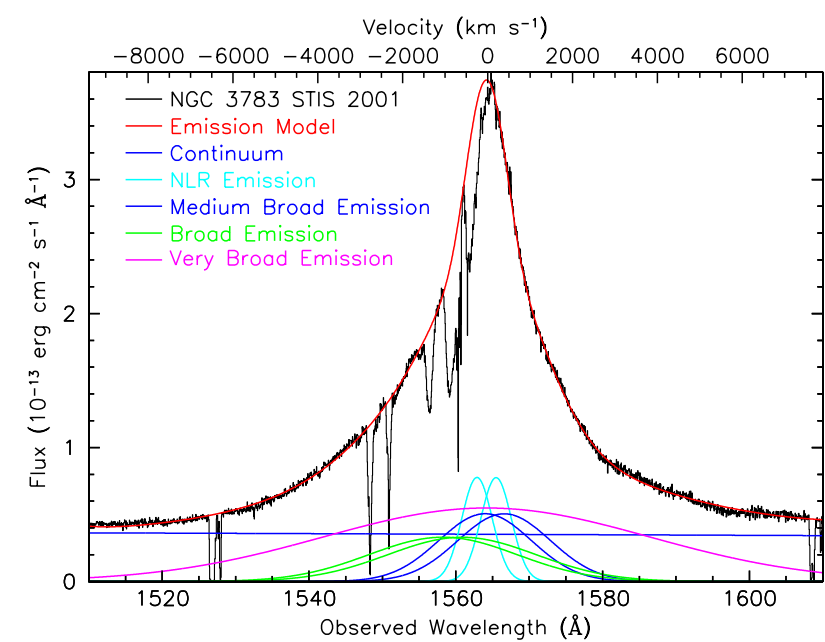

Fig. 2. Average STIS spectrum of the $\mathrm{C}$ IV region from all observations in the 2000-2001 campaign re-binned into $0.05 \AA$ pixels (black histogram). The solid red line tracing the data shows the total emission model. The key in the figure identifies the emission components in our model. The velocity scale along the top axis is for the blue component of the $\mathrm{C}$ IV doublet, $\lambda 1548.195$, relative to the host galaxy systemic redshift, $z=0.00973$ (Theureau et al. 1998).

major emission-line regions independently, as described below. For these individual regions, we froze the continuum parameters, free the line widths and fluxes first, and finally the line centers. Since the line centers, widths, and fluxes are free, we were not forcing any assumptions of symmetry on the line profiles; slight asymmetries in the line profiles can be accommodated by the differing centroids and widths of the various components. Once the major line-emitting regions have been fit $(\mathrm{Ly} \alpha+\mathrm{N} v, \mathrm{Si}$ Iv, $\mathrm{C}$ IV, and $\mathrm{He}$ II), we let all parameters vary freely to converge to a globally optimized best fit. In the sections below we separately describe in further detail our fitting process for the STIS 2001 spectrum first, and then the COS 2016 spectra.

\subsection{Modeling the unobscured STIS spectrum of NGC 3783}

To model the COS spectra of NGC 3783, we establish a baseline model by fitting the average STIS spectrum accumulated in the monitoring campaign from 2000-2001 that included STIS, FUSE, and Chandra observations (Gabel et al. 2003a; Kaspi et al. 2002). This spectrum has high $\mathrm{S} / \mathrm{N}$, and modeling it is straightforward, especially for C IV, since much of the line profile is uncontaminated by absorption. In this spectrum, after establishing preliminary continuum and line fluxes, we then fit the $\mathrm{C}$ IV profile in detail. This is the least-blended, highest $\mathrm{S} / \mathrm{N}$ emission feature. The complement of Gaussian widths and relative fluxes here serve as a starting point for the other major features. We then fit the $\mathrm{Ly} \alpha+\mathrm{N} v$ region, followed by Si IV and He II. After all regions have been individually optimized, we iterate on the final model of the full spectrum to obtain the best fit. The best-fit emission-line properties are given in Table A.2.

\subsubsection{Modeling the Unobscured C IV Profile}

Figure 2 shows the best fit, with the profiles of all the $\mathrm{C}_{\mathrm{IV}}$ emission components shown as well as the continuum level. We note that the best fit shown here is the final result following the global optimization to the full spectrum after all regions 


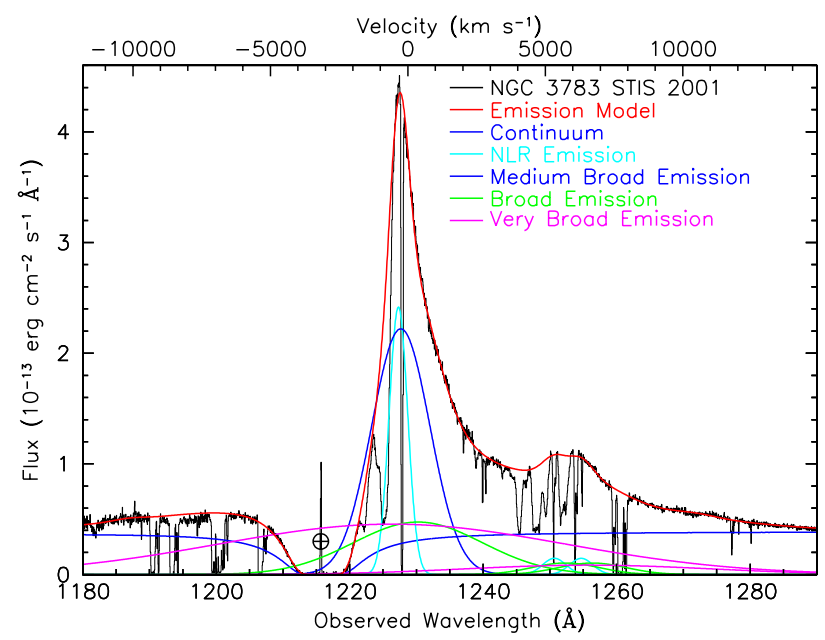

Fig. 3. Average STIS spectrum of the $\mathrm{Ly} \alpha+\mathrm{N} v$ region from all observations in the 2000-2001 campaign re-binned into $0.05 \AA$ pixels (black histogram). The solid red line tracing the data shows the total emission model. The key in the figure identifies the emission components in our model. The velocity scale along the top axis is for Ly $\alpha \lambda 1215.67$, relative to the host galaxy systemic redshift, $z=0.00973$ (Theureau et al. 1998). Geocoronal emission in the center of the Milky Way Ly $\alpha$ absorption trough is indicated with an Earth symbol. The solid blue line showing the continuum also illustrates the impact of the damped Milky Way $\operatorname{Ly} \alpha$ absorption. We note that the influence of this damping profile extends all the way from $1180 \AA$ on through the peak of Ly $\alpha$ in NGC 3783 and into the Nv profile at $1250 \AA$.

have been separately fit. The C IV narrow-line emission (cyan) is slightly blueshifted by about $-265 \mathrm{~km} \mathrm{~s}^{-1}$. The medium-broad emission (blue) and the very broad emission (magenta) lie near the systemic velocity. The broad components of the line profile (green) have a significant blue shift of $-900 \mathrm{~km} \mathrm{~s}^{-1}$.

\subsubsection{Modeling the unobscured Ly $\alpha$ profile}

The $\mathrm{Ly} \alpha+\mathrm{N} v$ region is more complicated due to the blending of the Ly $\alpha$ and $\mathrm{Nv}$ profiles and the strong impact of damped Ly $\alpha$ absorption from the Milky Way. Although we used the C IV profile as a guide and chose initial values for the Ly $\alpha$ components based on the $\mathrm{C}$ IV best fit, we do not constrain or tie these components to those determined for $\mathrm{C}$ IV. The final results do not wander far from these initial guesses, but we note that trial attempts with different plausible initial conditions also result in good fits that are slightly different; the blending and the foreground damped absorption lead to fits that are highly degenerate. Figure 3 illustrates the final fit to this region. As for $\mathrm{C}$ Iv, the narrow-line emission is significantly blue shifted, with a peak at $-340 \mathrm{~km} \mathrm{~s}^{-1}$. The medium-broad emission is also blueshifted at $-250 \mathrm{~km} \mathrm{~s}^{-1}$. In contrast to $\mathrm{C}_{\mathrm{IV}}$, the broad emission here is redshifted to $+350 \mathrm{~km} \mathrm{~s}^{-1}$ (for both Ly $\alpha$ and $\mathrm{Nv}$ ), but this may be biased due to the lack of a good view of the blue wing of Ly $\alpha$ and the blending of $\mathrm{N} v$ with $\operatorname{Ly} \alpha$.

\subsubsection{Modeling the unobscured Si IV profile}

Fitting the Si IV region is simpler since there is less contaminating intrinsic and foreground absorption. The line is fainter, however, and the $\mathrm{S} / \mathrm{N}$ is not as good as that in the $\mathrm{C}_{\text {IV }}$ and Ly $\alpha$ regions. Therefore an adequate fit requires only two narrow and two broad components for the Si Iv doublets, and a single very broad Si Iv base. Si Iv is also blended with several

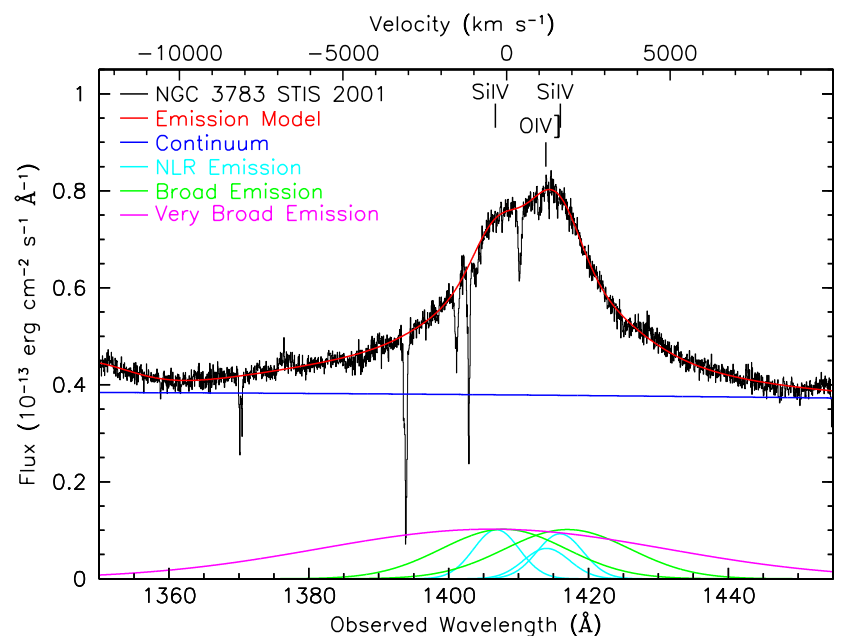

Fig. 4. Average STIS spectrum of the Si Iv region from all observations in the 2000-2001 campaign re-binned into $0.05 \AA$ pixels (black histogram). The solid red line tracing the data shows the total emission model. The key in the figure identifies the emission components in our model. The velocity scale along the top axis is for Si Iv $\lambda 1393.755$, relative to the host galaxy systemic redshift, $z=0.00973$ (Theureau et al. 1998).

O Iv] transitions; however, these appear to be weak, and only the cluster of the strongest transitions at $\lambda 1401$ is included in our model as an additional narrow component. The best fit is shown in Fig. 4. As with $\mathrm{C}_{\mathrm{IV}}$ and $\mathrm{Ly} \alpha$, the narrow lines are slightly blue shifted at $-100 \mathrm{~km} \mathrm{~s}^{-1}$, and, similar to $\mathrm{Ly} \alpha$, the broad lines are slightly redshifted at $+120 \mathrm{~km} \mathrm{~s}^{-1}$.

\subsubsection{Modeling the unobscured He II profile}

Finally, we show the fit to the He II region. This is important for establishing a good template for the broad-line region since He II $\lambda 1640$ is a recombination line to the excited $n=2$ state, and therefore unlikely to be affected by absorption ${ }^{2}$. It is also similar in ionization to $\mathrm{C}$ IV, and should form in the same physical regions and have similar overall kinematics. Since He II emission is faint, like Si IV, only three components are required: a narrow, a broad, and a very broad component. There is also slight contamination on the red wing by two $\mathrm{O}$ III] transitions, which we model with individual narrow components. Also note that the red half of the line center is strongly absorbed by foreground interstellar $\mathrm{Al}$ II $\lambda 1670$. Finally, this whole complex sits atop the far red wing of the much brighter $C_{\text {IV }}$ emission line. Figure 5 shows the best fit to the He II region. As for all previous emission lines, the narrow-line component is slightly blue-shifted at $-150 \mathrm{~km} \mathrm{~s}^{-1}$; the broad component is significantly blue-shifted at $-520 \mathrm{~km} \mathrm{~s}^{-1}$, but not as strongly as C IV.

\subsection{Modeling the average obscured COS spectrum of NGC 3783}

Once we had a good baseline model for the emission spectrum of NGC 3783, we can look at how the spectrum has changed from the unobscured state in 2001 to the obscured state that

\footnotetext{
2 He II $n=2$ is $48 \mathrm{eV}$ above the ground state, and maintaining a significant population of ions in this state requires high temperatures $\left(T \gtrsim 6 \times 10^{5} \mathrm{~K}\right)$, or high densities $\left(n \gtrsim 10^{15} \mathrm{~cm}^{-3}\right)$ neither of which are typical of BLR conditions of $T \sim 10^{4} \mathrm{~K}$ and $n \sim 10^{10} \mathrm{~cm}^{-3}$ (Osterbrock \& Ferland 2006).
} 


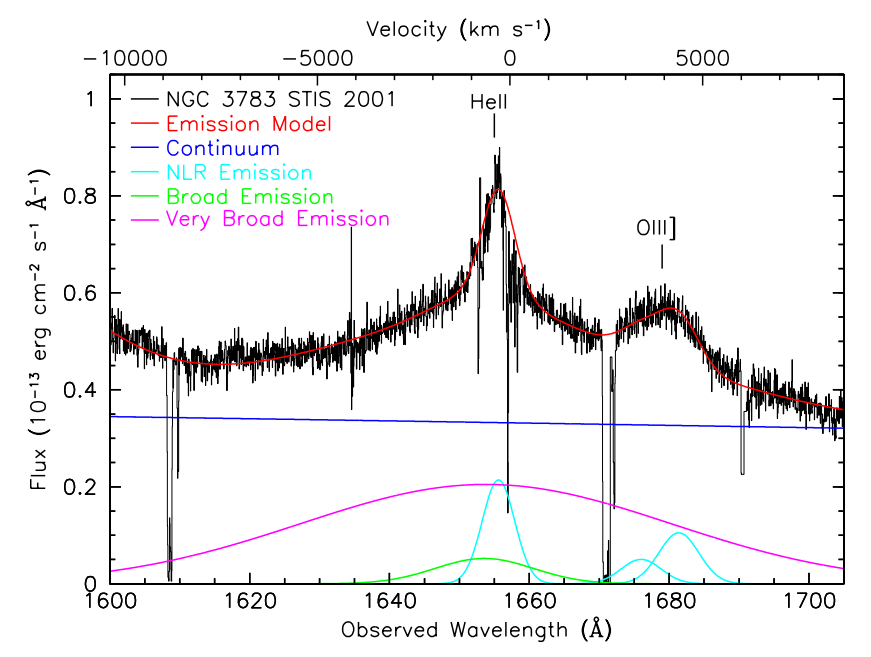

Fig. 5. Average STIS spectrum of the He II region from all observations in the 2000-2001 campaign re-binned into $0.05 \AA$ pixels (black histogram). The solid red line tracing the data show the total emission model. The key in the figure identifies the emission components in our model. The velocity scale along the top axis is for He II $\lambda 1640.45$, relative to the host galaxy systemic redshift, $z=0.00973$ (Theureau et al. 1998). All narrow absorption features are foreground ISM lines.

appears in our 2016 observation. We first fit the unweighted average sum of the two spectra from 2016-12-12 and 201612-21. As for the STIS spectrum, we fit the obscured COS selected regions at a time, beginning with $\mathrm{He}$ II, which we do not expect to be absorbed, followed by $\mathrm{C}_{\mathrm{IV}}$, Ly $\alpha+\mathrm{N} v$, and then $\mathrm{Si}$ iv. After all regions were individually optimized, we iterated on the final model of the full spectrum to obtain the best fit. Table A. 2 compares the best-fit emission-line properties for the average obscured COS spectrum in 2016 to the STIS spectrum from 2000-2001. Once we have this fit to the average spectrum, we then scaled the model to adjust the continuum and line fluxes to the levels of the individual observations on 2016-1212 and 2016-12-21, and then optimized the fits to each of these individual spectra. Results for the two separate observations are given in Table A.3. In the following sections, we describe the fits to each of the individual regions in the obscured COS spectrum.

\subsubsection{Modeling the obscured He II profile}

Since we do not expect the He II emission line to be absorbed, we began our fits with that line to see what intrinsic changes in the profile of the broad emission lines may have taken place between 2001 and 2016. In Fig. 6 we compare the STIS 2001 spectrum to the COS 2016 spectrum. The continuum and the underlying emission of the $\mathrm{C}$ IV profile have been subtracted. The STIS spectrum is scaled up by a factor of 1.2 to match the flux in the high-velocity wings. $\left.\mathrm{O}_{\mathrm{III}}\right]$ emission that sits on the red wing of He II has not been removed. One can see that overall, there is little change in the He II profile. Interestingly, the narrow component has moved redwards and now lies at the systemic velocity. To optimize the model to the COS spectrum, we adjust the line fluxes in the model and the wavelength of the narrow component accordingly before letting the fit iterate to a new minimum. The resulting best fit is shown in Fig. 6 .

To compare the broad-line region profiles more closely, we subtract the modeled narrow emission lines from each profile and show the result in Fig. 7. Here we see that the STIS and COS

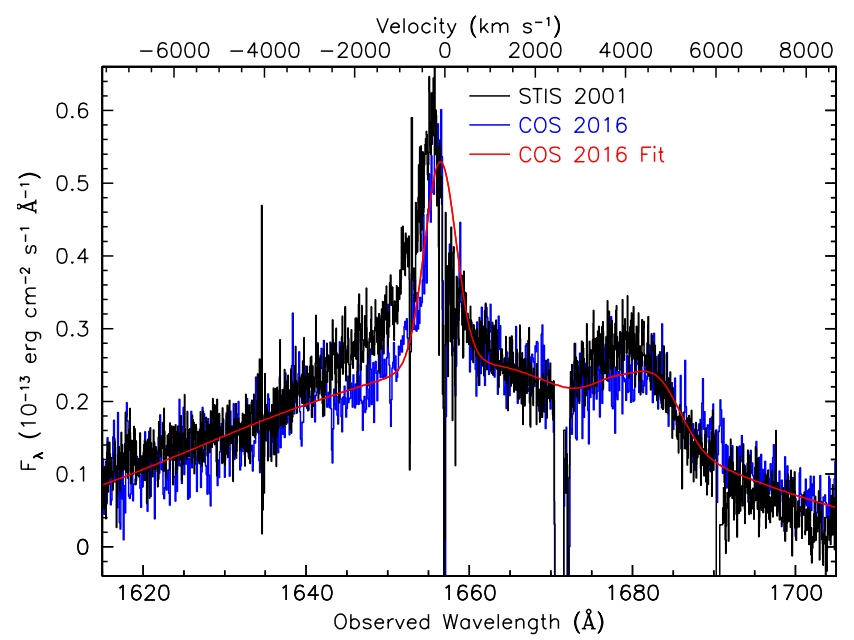

Fig. 6. Comparison of STIS and COS spectra of the region surrounding the He II emission line. The black histogram shows the average STIS 2001 spectrum. The blue histogram shows the unweighted average COS spectrum from the 12 December 2016 and 21 December 2016 observations. Both spectra have the continuum subtracted as well as the underlying emission of the $C_{\text {IV }}$ emission line. The STIS spectrum is scaled up by $1.2 \times$ to match the flux in the far wings of the COS spectrum. The solid red line is the best-fit emission model for the COS spectrum. The velocity scale along the top axis has zero velocity for He II $\lambda 1640.45$ at the host-galaxy redshift of 0.00973 (Theureau et al. 1998). All narrow absorption features are foreground ISM lines.

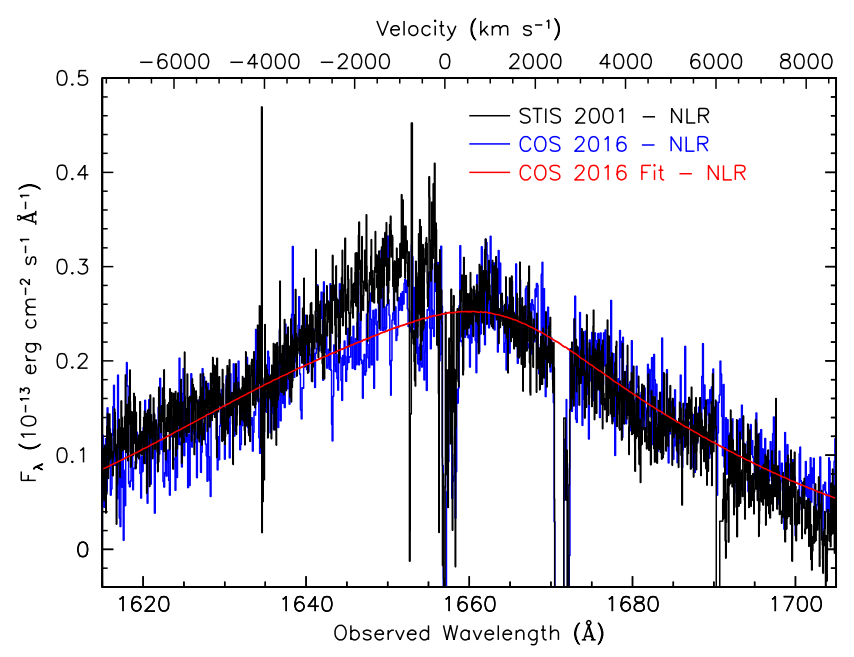

Fig. 7. Comparison of STIS and COS spectra of the region surrounding the He II emission line omitting the narrow emission. The black histogram shows the average STIS 2001 spectrum with the narrow components of $\mathrm{He}_{\mathrm{II}}$ and $\mathrm{O}_{\mathrm{III}}$ ] subtracted. The blue histogram shows the unweighted average COS spectrum from the 12 December 2016 and 21 December 2016 observations, also with the narrow components subtracted. Both spectra have the continuum subtracted as well as the underlying emission of the C IV emission line. The STIS spectrum is scaled up $1.2 \times$ to match the flux in the far wings of the COS spectrum. The solid red line represents the best-fit emission model for the COS spectrum. The velocity scale along the top axis has zero velocity for He II $\lambda 1640.45$ at the host-galaxy redshift of 0.00973 (Theureau et al. 1998).

profiles are very similar, except for a little added emission in the STIS spectrum on the blue side of line center. Although the STIS spectrum exhibits this excess emission relative to COS, there is no indication of broad absorption in the COS spectrum; the 


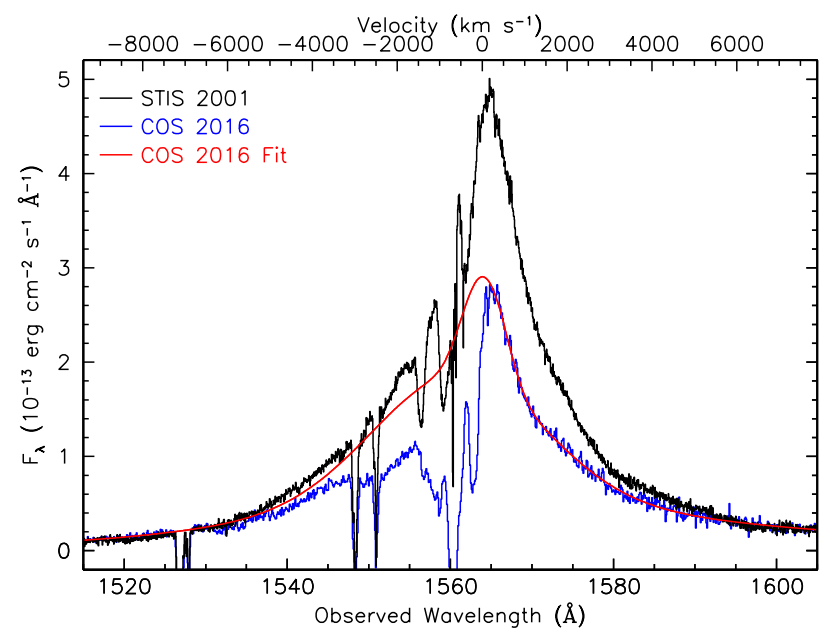

Fig. 8. Average STIS spectrum of the C IV region from all observations in the 2000-2001 campaign re-binned into $0.05 \AA$ pixels (black histogram). The blue histogram shows the average COS spectrum from 2016. The STIS data have been scaled up by a factor of 1.45 so that the intensities match in the broad wings. The solid red line tracing the data is the total emission model for the average 2016 COS spectrum. The velocity scale along the top axis is for $C_{\text {IV }} \lambda 1548.195$, relative to the host galaxy systemic redshift, $z=0.00973$ (Theureau et al. 1998).

differences between the two profiles are accommodated merely by adjusting the flux and wavelength of the broad component (now shifted to the red as for the narrow component). Table A.2 shows that the flux in the broad component of the COS spectrum is $17 \%$ lower compared to STIS, and shifted $1760 \mathrm{~km} \mathrm{~s}^{-1}(10 \AA)$ to the red. The fitted line profile passes smoothly through all the data in both line wings.

\subsubsection{Modeling the obscured C IV profile}

From our fit to the He II profile, we expect that our basic model for C IV should also be very similar in the COS 2016 spectrum compared to the STIS 2001 spectrum. Figure 8 compares the CIV profile from STIS 2001 to COS 2016. As for He II, both spectra have been continuum subtracted, and the STIS spectrum has been scaled up by a factor of 1.45 so that the intensities match in the broad wings beyond $\pm 6000 \mathrm{~km} \mathrm{~s}^{-1}$. For reference, for COS 2016 the continuum flux $F(1560 \AA)=7.5 \times 10^{-14} \mathrm{erg} \mathrm{cm}^{-2} \mathrm{~s}^{-1} \AA^{-1}$; for STIS 2011, $F(1560 \AA)=3.5 \times 10^{-14} \mathrm{erg} \mathrm{cm}^{-2} \mathrm{~s}^{-1} \AA^{-1}$. We note that even though the continuum in 2016 is more than twice as bright as in 2001, the C IV emission line is fainter, particularly in the mid section of the profile. However, the narrow cores of each profile are nearly unchanged. Indeed, in our best fit, the narrow-line component has the same velocity and width, and is within $10 \%$ of the flux observed in 2001.

Given the apparently fixed intensity of the narrow-line emission, Fig. 9 compares the profiles if we subtract this fitted emission component from the profiles of each spectrum, and from the best fit curve. One can now see that most of the excess emission in the 2001 spectrum is in what we identified as the mediumbroad component in Fig. 2, which are the blue curves near a systemic velocity of zero in that figure. We note that this component is not needed in our fit to either He II emission profile.

If we now remove this medium-broad component from the STIS spectrum and compare the result to the observed profile in 2016, Fig. 10 shows that the shape of the two profiles match well in both the far wings of the line (both red and blue), and on the

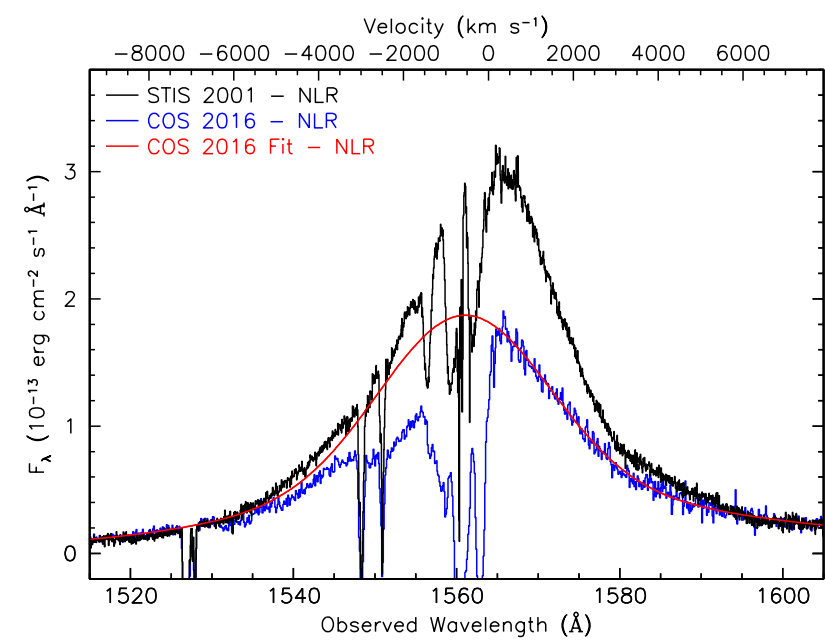

Fig. 9. Average STIS spectrum of the $C_{\text {IV }}$ region from all observations in the 2000-2001 campaign re-binned into $0.05 \AA$ pixels (black histogram) with the narrow-line component of the fit subtracted from the data. The blue histogram shows the average COS spectrum from 2016 with the narrow-line component subtracted. The STIS data have been scaled up by a factor of 1.45 so that the intensities match in the broad wings. The solid red line tracing the data is the total emission model for the average 2016 COS spectrum with the narrow-line component removed. The velocity scale along the top axis is for $C_{\text {IV }} \lambda 1548.195$, relative to the host galaxy systemic redshift, $z=0.00973$ (Theureau et al. 1998).

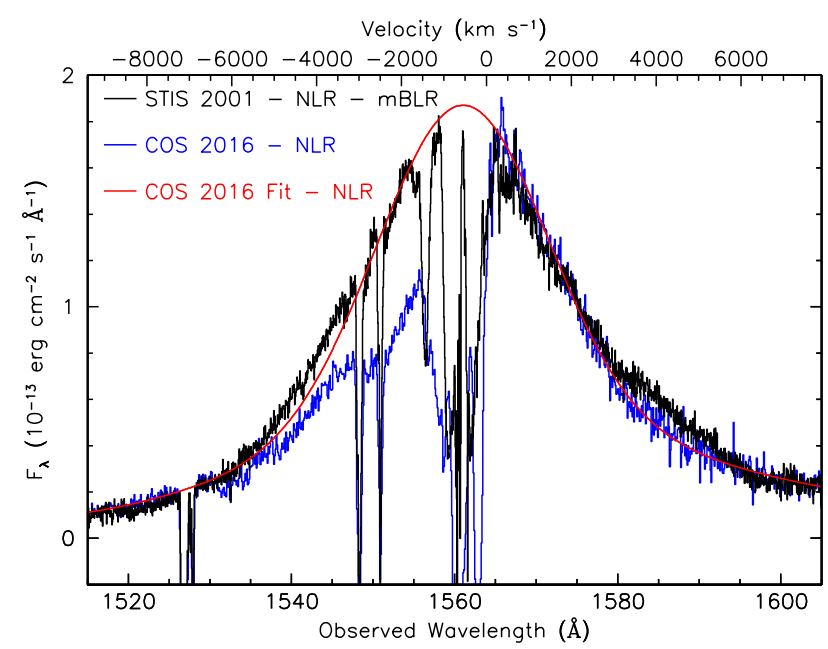

Fig. 10. Average STIS spectrum of the C Iv region from all observations in the 2000-2001 campaign re-binned into $0.05 \AA$ pixels (black histogram) with both the narrow-line and medium-broad components of the fit subtracted from the data. The blue histogram shows the average COS spectrum from 2016 with the narrow-line and medium-broad components subtracted. The STIS data have been scaled up by a factor of 1.45 so that the intensities match in the broad wings. The solid red line tracing the data is the total emission model for the average 2016 $\mathrm{COS}$ spectrum with the narrow-line component removed. The velocity scale along the top axis is for C Iv $\lambda 1548.195$, relative to the host galaxy systemic redshift, $z=0.00973$ (Theureau et al. 1998).

red side of the line. The minor differences in the profiles on the red side of line center are accommodated by slight adjustments in the relative fluxes of the broad and very broad components from 2001 to 2016 . However, there is a noticeable deficiency in flux on the blue side of the line profile, which we interpret as absorption due to the appearance of the obscurer. 


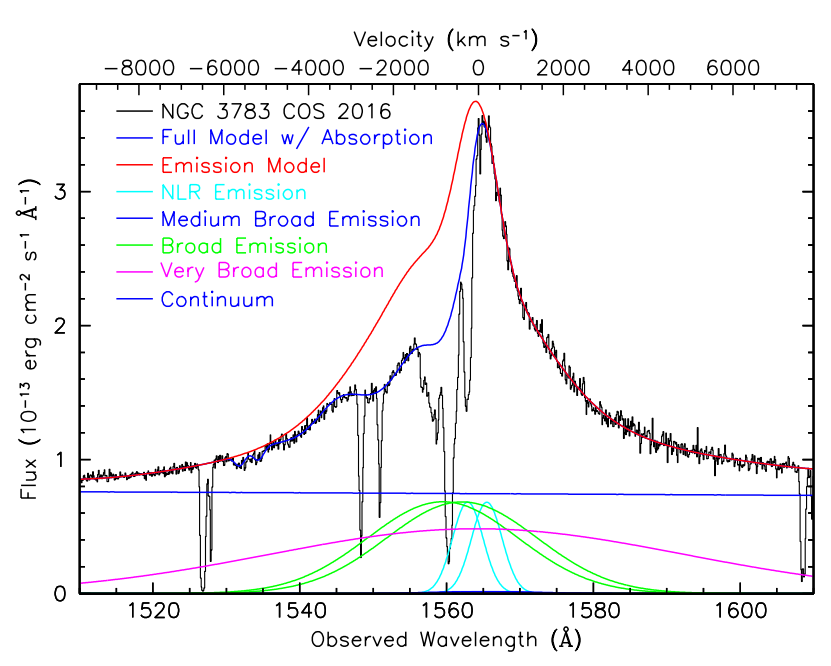

Fig. 11. Average COS spectrum of the $\mathrm{C}$ Iv region from observations in 2016 December (black histogram). The solid red line tracing the data is the total emission model. The solid blue line tracing the data is the emission model modified by components that model the broad absorption. The key in the figure identifies the emission components in our model. The velocity scale along the top axis is for the blue component of the $C_{\text {IV }}$ doublet, $\lambda 1548.195$, relative to the host galaxy systemic redshift, $z=0.00973$ (Theureau et al. 1998).

So, to adapt the Civ profile from 2001 to what we observe in 2016, we fixed the intensity of the narrow-line components at the 2001 value, scaled the intensity of the very broad emission up by the factor of 1.45 required to match the far wings, kept all line centers and widths fixed, and allowed the mediumbroad and broad fluxes to vary freely. As a result, the mediumbroad component's intensity dropped to nearly zero, with most remaining flux in the broad component. We then freed all parameters (all line centers, widths and intensities) and optimized the fit. Figure 11 shows the best-fit emission model and all its components.

To provide a semi-empirical characterization of the blueshifted absorption in the $C_{\text {IV }}$ profile, we added a series of $C_{\text {IV }}$ absorption-line doublets that coincide with the deepest inflection points in the blue wing at outflow velocities of $-2590,-4625$, -5515 , and $-6025 \mathrm{~km} \mathrm{~s}^{-1}$. These doublets are modeled as Gaussians in optical depth with relative velocities linked at the ratio of rest wavelengths, identical widths of $\sim 500 \mathrm{~km} \mathrm{~s}^{-1}$ for each doublet component, and blue to red optical depths assumed to have a 2:1 ratio. This characterization enables us in our subsequent modeling to test whether similar absorption is present in other lines such as $\operatorname{Ly} \alpha, \mathrm{Nv}$, and Si Iv. The full final model including these additional absorption components is also shown in Fig. 11.

Given that there are changes in the $\mathrm{C}$ IV profile that do not strictly mimic our comparison of the He II profiles, one might legitimately ask whether the COS 2016 C IV profile could be modeled without invoking absorption components, but allowing for more dramatic changes in the emission-line structure. To test this possibility, we made interactive adjustments to the line centers, widths, and fluxes of the narrow, mediumbroad, broad, and very broad components in the C IV profile. We also included the portions of the line profile in the 1530 $1545 \AA$ and $1552-1555 \AA$ regions which we had been treating as absorbed and required them to be part of the emission profile. We then let the fit try to optimize itself to the observed profile without including any absorption. Figure 12 shows the resulting fit.

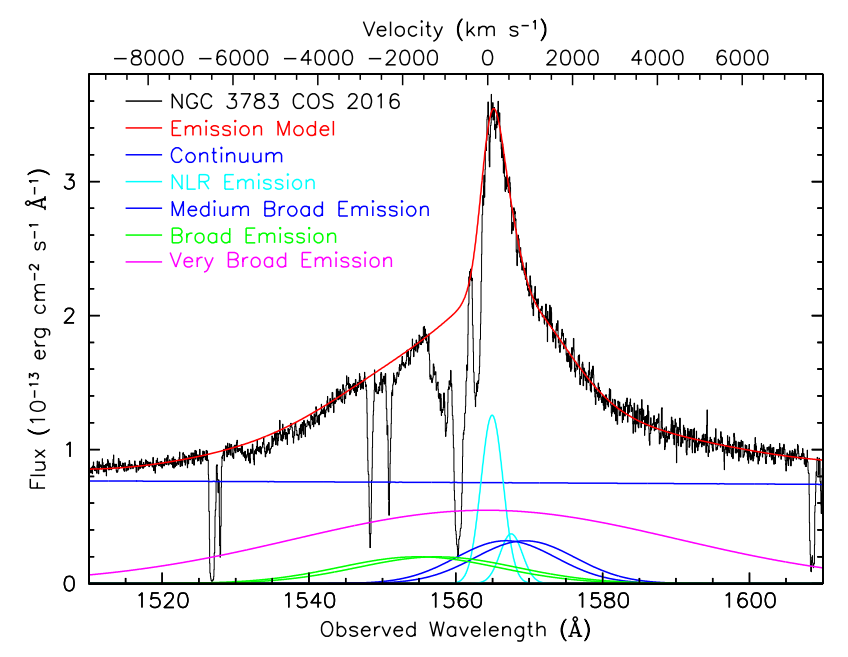

Fig. 12. Best fit with no absorption to the average COS spectrum of the C IV region from observations in 2016 December. The black histogram shows the data. The solid red line tracing the data is the total emission model. The key in the figure identifies the emission components in our model. The velocity scale along the top axis is for the blue component of the C Iv doublet, $\lambda 1548.195$, relative to the host galaxy systemic redshift, $z=0.00973$ (Theureau et al. 1998).

This alternative interpretation of the line profile is not a good fit, and it exhibits several unsatisfactory features. First, there are smaller-scale, shallow features on the blue side of the line profile in the -2000 to $-6000 \mathrm{~km} \mathrm{~s}^{-1}$ velocity range that are not fit well; these regions still look like shallow absorption features. Second, the narrow emission components have to move in velocity to $+100 \mathrm{~km} \mathrm{~s}^{-1}$, and they have an unphysical ratio of $3.4: 1$ for the blue to red intensity ratio, which should be $\leq 2: 1$. Finally, the medium-broad components have moved in velocity from near systemic in the STIS spectrum to $+550 \mathrm{~km} \mathrm{~s}^{-1}$ here, and the broad components have blue-shifted even more to $-1650 \mathrm{~km} \mathrm{~s}^{-1}$.

As we noted earlier, the He II profile does not show such dramatic changes. We have tried to correct some of these shortcomings by fixing the narrow emission components in flux and velocity at the STIS 2001 values, forcing the medium-broad components to have zero systemic velocity (as they did in the STIS spectrum), and then let everything else vary freely. As expected, the fit is even worse, and the irregular features on the blue wing of the line profile simply cannot be accommodated by such a model. We conclude that absorption is the best explanation for the changes in the shape of the C IV profile.

\subsubsection{Modeling the obscured Ly $\alpha$ profile}

To model the Ly $\alpha$ and $\mathrm{N} v$ region of the COS spectrum, we began with the emission model developed for the STIS 2001 spectrum. As with $\mathrm{C}_{\mathrm{IV}}$, we kept the narrow emission components fixed in flux, velocity, and width. We scaled the broader components down in flux to accommodate the decrease in overall flux. Given the convincing evidence for broad, blue-shifted absorption affecting the $\mathrm{C}$ IV emission-line profile during the obscured state of NGC 3783, we then imposed a scaled replica of the modeled $\mathrm{C}$ IV absorption to the the Ly $\alpha$ and $\mathrm{N} v$ regions. This initial model for the absorption has the same velocities, widths, and optical depths as for $\mathrm{C} \mathrm{IV}$, and provides a very good approximation to the new features present on the red wing of $\operatorname{Ly} \alpha$ (that are due to $\mathrm{Nv}$ absorption) as well as the more subtle changes in the blue 


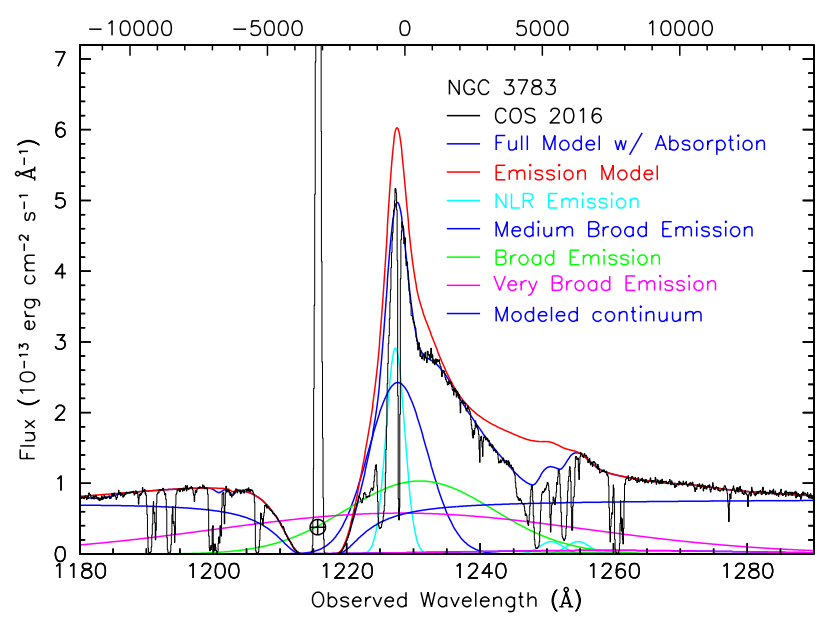

Fig. 13. Average COS spectrum of the $\mathrm{Ly} \alpha+\mathrm{N} v$ region from observations in 2016 December (black histogram). The solid red line tracing the data is the total emission model. The solid blue line tracing the data is the emission model modified by components that model the broad absorption. The key in the figure identifies the emission components in our model. Geocoronal emission in the center of the Milky Way Ly $\alpha$ absorption trough is indicated with an Earth symbol. The velocity scale along the top axis is for $\operatorname{Ly} \alpha \lambda 1215.67$, relative to the host galaxy systemic redshift, $z=0.00973$ (Theureau et al. 1998).

wing of Ly $\alpha$. After selecting initial values by eye that give an approximate fit, we then start freeing parameters and iterating to an overall solution as described at the beginning of Sect. 3 . For our final fit, all parameters varied freely. Figure 13 shows the resulting best fit to the $\mathrm{Ly} \alpha$ and $\mathrm{N} v$ region. The final model for the Ly $\alpha$ absorption profile resembles the $\mathrm{C}$ iv profile closely in velocity and width (see Sect. 3.3), but it is shallower in optical depth.

\subsubsection{Modeling the obscured Si IV profile}

Modeling the Si Iv region is important. Since, of the species that show significant absorption it is the one with the lowest ionization, it sets a lower bound on the ionization state of the broad absorber. Unlike in NGC 5548 (Arav et al. 2015), absorption from lower ionization species such as $\mathrm{Si}$ II or $\mathrm{C}_{\text {II }}$ are not present in our spectra. Fits to the Si Iv region, however, are more ambiguous since the line is not very bright, and the $\mathrm{S} / \mathrm{N}$ is not as good as in the $\mathrm{C}$ IV and Ly $\alpha$ regions. Si IV is also blended with several $\mathrm{O}_{\mathrm{IV}}$ ] transitions; however, these appear to be weak, and as in the STIS spectrum, they primarily affect rest wavelengths $>1403 \AA$. Starting again with the model for the STIS 2001 spectrum, in Fig. 14 we compare the STIS and COS continuum-subtracted line profiles. No scaling has been applied to either spectrum. Again, we have the remarkable result that despite the continuum being twice as bright in 2016 as in 2001, the emission line in 2001 is slightly brighter. As with C Iv, most of this brighter emission is in the core of the line.

As for C IV, next we subtract the narrow emission components of Si IV and O Iv]. Figure 15 shows this net spectrum for both epochs. A slight excess still remains near the line center. Recall that we use only three Gaussians to model Si IV since it is so weak. This remaining excess present in the STIS spectrum is analogous to what we have modeled as medium-broad emission in the C IV profile. Removing this would then bring the STIS spectrum almost into line with the model fit to the broad plus very broad emission in the COS 2016 spectrum.

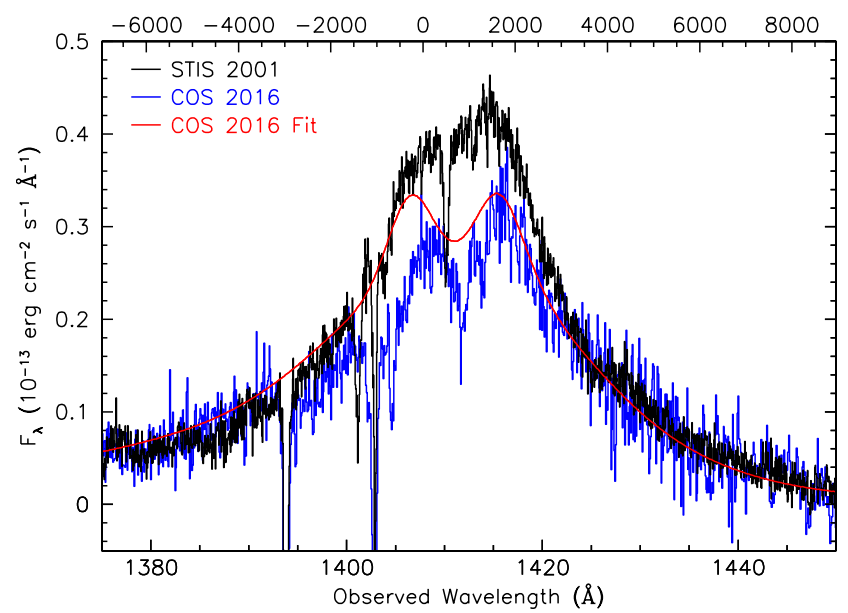

Fig. 14. Comparison of STIS and COS spectra surrounding the region of the Si Iv emission line. The black histogram shows the average STIS 2001 spectrum. The blue histogram is the unweighted average COS spectrum from 2016. The solid red line is the best-fit emission model for the COS spectrum. The velocity scale along the top axis is for Si IV $\lambda 1393.755$, relative to the host galaxy systemic redshift, $z=0.00973$ (Theureau et al. 1998).

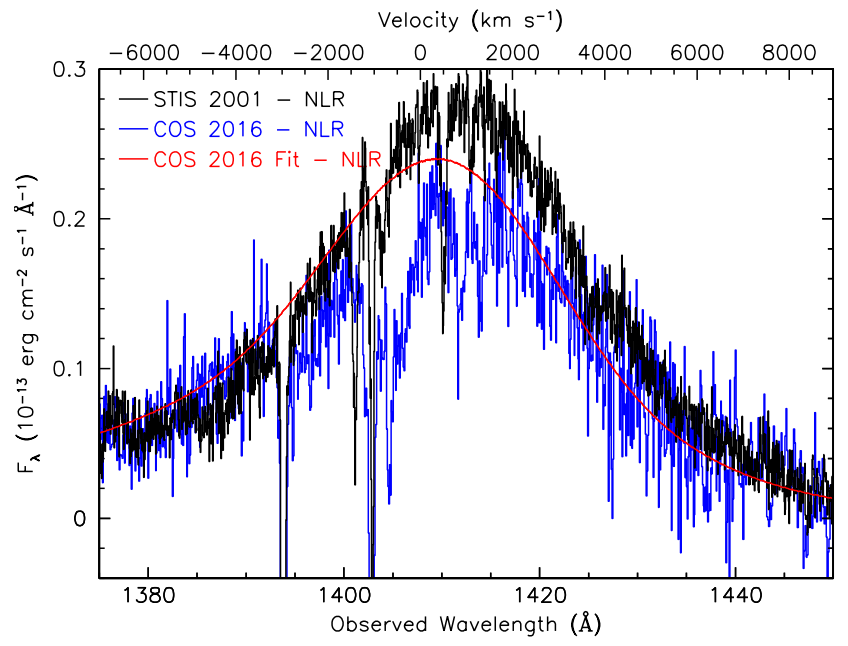

Fig. 15. Comparison of STIS and COS spectra of the region surrounding the Si Iv emission line omitting the narrow emission components. The black histogram shows the average STIS 2001 spectrum with the narrow emission components of Si IV and OIV] subtracted. The blue histogram is the unweighted average COS spectrum from 2016, also with the narrow emission components subtracted. The solid red line is the best-fit emission model for the COS spectrum minus the narrow emission components. The velocity scale along the top axis is for Si Iv $\lambda 1393.755$, relative to the host galaxy systemic redshift, $z=0.00973$ (Theureau et al. 1998).

We now investigate whether there is broad absorption present in the COS 2016 spectrum. Near the line peak this is uncertain since the narrow absorption lines are deeper and stronger than in 2001, and they may be responsible for most of the diminution in flux we observe. However, at velocities in the blue wing from $-1500 \mathrm{~km} \mathrm{~s}^{-1}$ to $-3000 \mathrm{~km} \mathrm{~s}^{-1}$, there is an obvious depression in flux below the model profile. Smoothing both spectra with a seven-pixel running boxcar filter as shown in Fig. 16 shows this depression more clearly. The model, however, is far from unique. Given the $\mathrm{S} / \mathrm{N}$, an acceptable fit is possible where the only absorption is due to enhanced absorption in the narrow absorption lines. The broad absorption shown in Figs. 15 and 16 should therefore be considered upper limits. Relative to the continuum, 


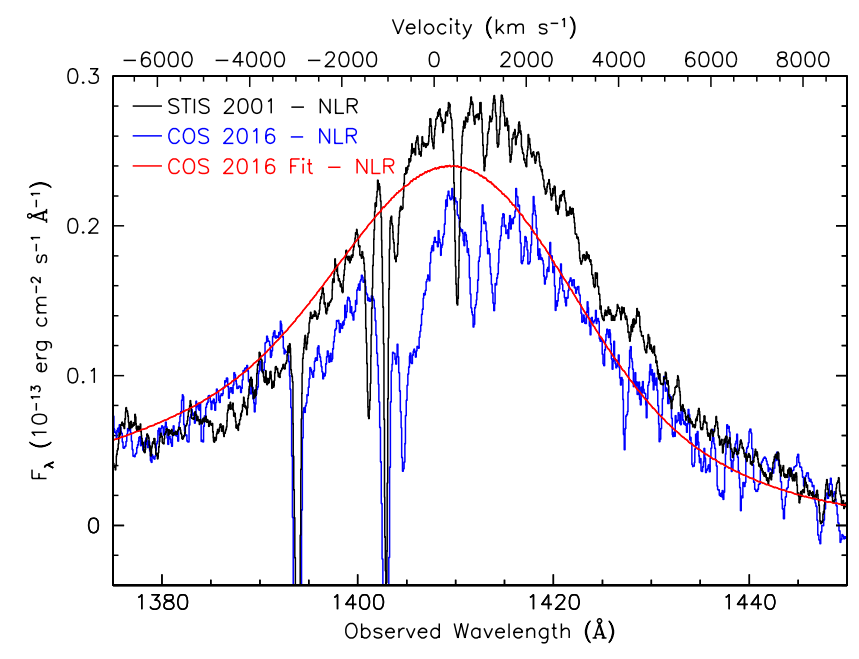

Fig. 16. Spectra of the Si IV region as in Fig. 15, but with both the STIS and the COS spectra smoothed by a seven-pixel running boxcar filter. The solid red line is the best-fit emission model for the COS spectrum minus the narrow emission components. The velocity scale along the top axis is for Si Iv $\lambda 1393.755$, relative to the host galaxy systemic redshift, $z=0.00973$ (Theureau et al. 1998).

the depression in flux at $-2500 \mathrm{~km} \mathrm{~s}^{-1}$ corresponds to an optical depth of only 0.05 .

\subsubsection{Modeling the obscured $\mathrm{H} \beta$ profile}

Similarly to $\mathrm{He}$ II, $\mathrm{H} \beta$ emission originates as recombination radiation to an excited level. Likewise, we do not expect absorption in such a feature. As our baseline for comparison, we used a STIS spectrum obtained in 2011 when NGC 3783 was in an unobscured state. Our FEROS spectrum was obtained on 201612-12, simultaneously with our first XMM-Newton spectrum of NGC 3783 in its obscured state. Analogous to our fit to the C IV region, we fit both the STIS and the FEROS $\mathrm{H} \beta$ spectra using a power law for the continuum, a narrow emission component, a medium-broad component, a broad component, and a very broad component. Significantly, both spectra also require an additional emission bump on the red side of the line profile, although the bump is more prominent in the FEROS spectrum. Figure 17 compares the unobscured STIS spectrum of the $\mathrm{H} \beta$ region to the FEROS spectrum. As in our previous comparisons, we have subtracted the continuum and scaled the FEROS spectrum to match the flux level in the far blue wing of the emission-line profile.

In Fig. 18, we compare the profiles with both the continuum and the narrow emission component subtracted. The two profiles now show behavior similar to our previous emission-line comparisons - the core of the emission line in the unobscured state is brighter. In the obscured state, this core seems to have faded, analogous to the disappearance of the medium-broad component in C IV. This makes the red emission bump in the $\mathrm{H} \beta$ line profile more prominent in the obscured state. These changes can be accommodated simply by changing the relative fluxes of the emission components. There is no need for any absorption in the line profile. All the components in the final best-fit to the FEROS $\mathrm{H} \beta$ emission line profile are illustrated in Fig. 19.

\subsection{Photoionization modeling of the broad absorption lines}

Our models of the emission and broad absorption in NGC 3783 allow us to measure the physical properties of the gas causing

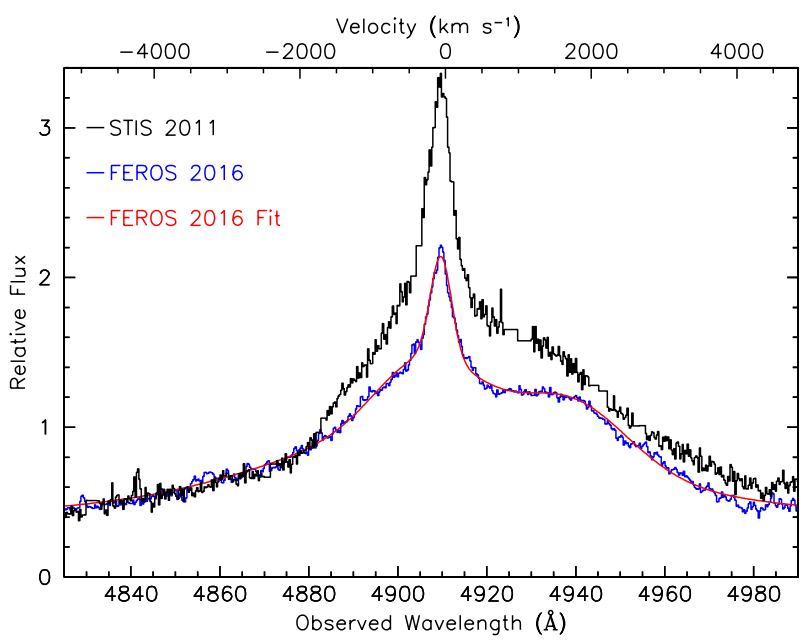

Fig. 17. FEROS spectrum of the $\mathrm{H} \beta$ region from observations in 2016 December (blue histogram) compared to the STIS spectrum of 2011 (black). The continuum has been subtracted from each spectrum, and the FEROS spectrum has been scaled to match the flux in the far blue wing of the STIS spectrum. The solid red line tracing the FEROS data is the total emission model with the continuum subtracted. The velocity scale along the top axis is for the rest wavelength of $\mathrm{H} \beta$ relative to the host galaxy systemic redshift, $z=0.00973$ (Theureau et al. 1998).

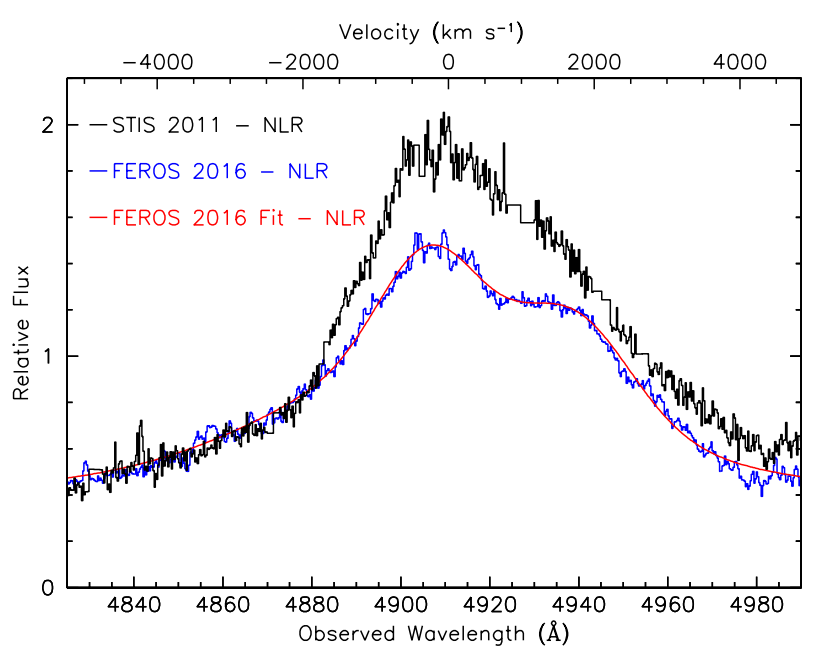

Fig. 18. FEROS (blue) and STIS (black) spectra of the $\mathrm{H} \beta$ region with the continuum and narrow $\mathrm{H} \beta$ emission component subtracted from each. The FEROS spectrum has been scaled to match the STIS spectrum in the far blue wing of the emission line. The solid red line tracing the data shows the total emission model with the continuum and narrow emission component subtracted. The velocity scale along the top axis is for the rest wavelength of $\mathrm{H} \beta$ relative to the host galaxy systemic redshift, $z=0.00973$ (Theureau et al. 1998).

the broad absorption and possibly also the soft X-ray obscuration observed in 2016 December. Using our model for the emission spectrum, we produce normalized spectra for the regions affected by the broad UV absorption, as shown in Fig. 20.

We were able to integrate our normalized models for the broad absorption using the apparent optical depth method of Savage \& Sembach (1991) to obtain column densities, $N_{\text {ion }}$. However, since the lines appear to be saturated, these column densities are only lower limits to the true column density. In Table 1 we summarize the observable properties of the modeled broad absorption troughs. The equivalent width (EW) is integrated from the normalized spectra between the velocity limits 


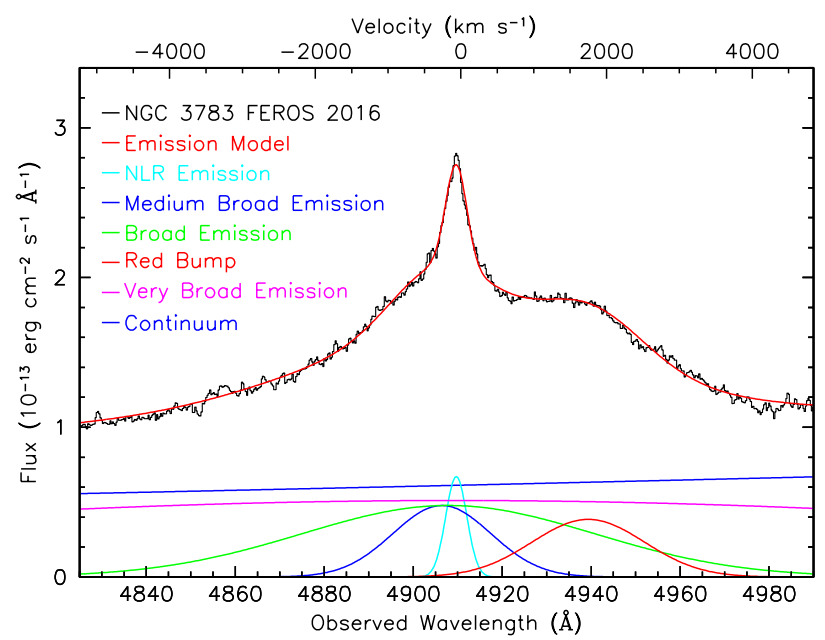

Fig. 19. FEROS spectrum of the $\mathrm{H} \beta$ region from observations in 2016 December (black histogram). The solid red line tracing the data is the total emission model. The key in the figure identifies the emission components in our model. The velocity scale along the top axis is for the rest wavelength of $\mathrm{H} \beta$ relative to the host galaxy systemic redshift, $z=0.00973$ (Theureau et al. 1998).

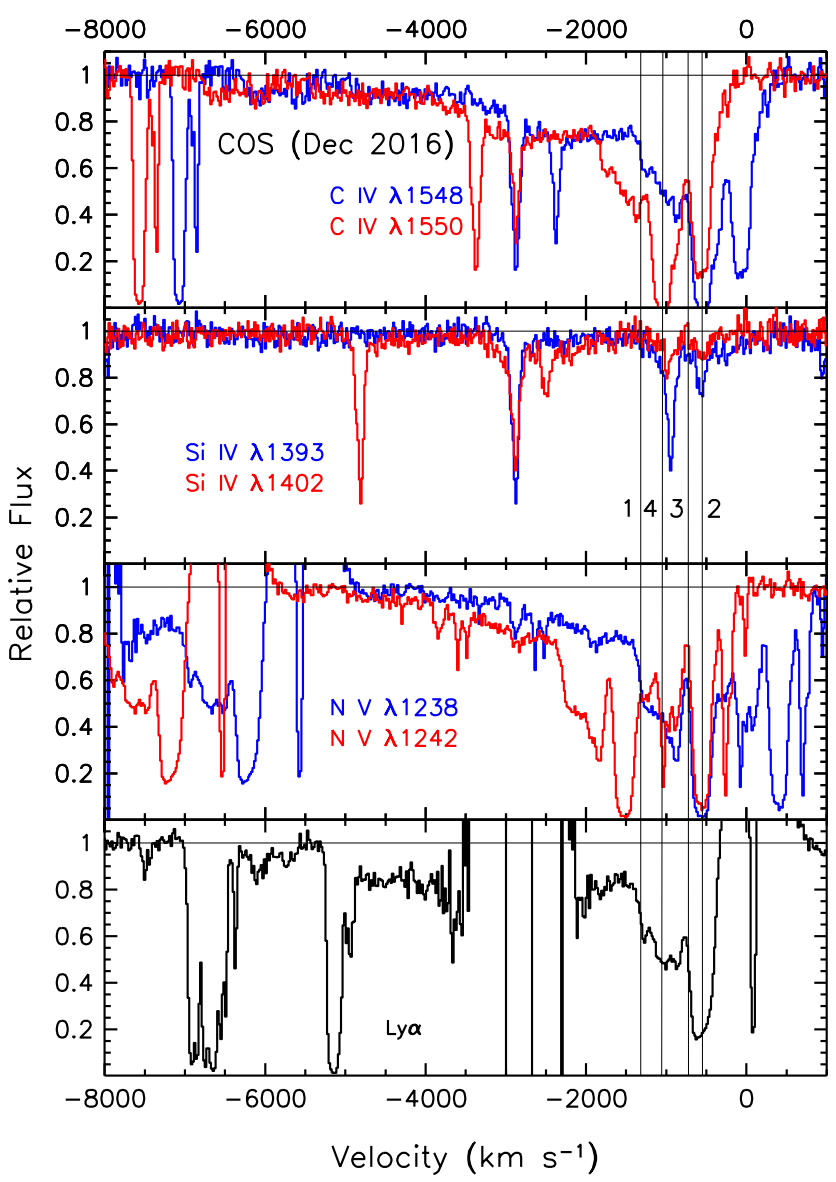

Fig. 20. Normalized spectra of the broad absorption features in NGC 3783 (2016-12-12). The velocities of Components 1-4 as given by Gabel et al. (2003b) are marked by thin vertical blue lines and labeled.

$v_{1}$ and $v_{2}$. The deepest point of the trough is at velocity $v_{0}$, and we use the dispersion $\sigma_{v}$ to characterize the width of the trough. The covering fraction, $C_{\mathrm{f}}$, is measured by assuming that the profile is saturated at the deepest point of the trough.
Unlike the obscured state of NGC 5548 (Kaastra et al. 2014; Arav et al. 2015), where absorption from low-ionization species such as $\mathrm{C}_{\text {II }} \lambda 1335$ and $\mathrm{Si}$ III $\lambda 1206$ was present, in the obscured state of NGC 3783 the lowest ionization broad absorption feature is Si Iv. To model the ionization state of the absorbing clouds, we used the unobscured spectral energy distribution (SED) of NGC 3783 as presented in the top panel of Fig. 6 of Mehdipour et al. (2017). We derive the ionization balance of the obscurer using a grid of models generated using the Cloudy v17.00 photoionization code (Ferland et al. 2017). Our grid covers a range in ionization parameter $\log \xi\left(\mathrm{erg} \mathrm{cm} \mathrm{s}^{-1}\right)$ from 1.0 to 2.5, and total column density $\log N_{\mathrm{H}}\left(\mathrm{cm}^{-2}\right)$ from 21.5 to 23.7. The ionization parameter has the usual definition, $\xi=L_{\mathrm{ion}} /\left(n r^{2}\right)$, where $L_{\text {ion }}$ is the ionizing luminosity from 1 to $1000 \mathrm{Ryd}, n$ is the density, and $r$ is the distance of the absorbing cloud from the ionization source.

For absorption troughs detected in our spectra, Ly $\alpha, \mathrm{Nv}$, $\mathrm{C}$ IV, and Si Iv, we used the lower limits on the total column density given in Table 1 and show these limits as solid lines in Fig. 21. Using a transmission profile with the same shape in velocity as $\mathrm{C}$ IV, we allow the optical depths at the locations of $\mathrm{C}_{\text {II }} \lambda 1335$ and Si II $\lambda 1260$ to vary until $\chi^{2}$ increases by 4.0 above its minimum value. This then gives us $2 \sigma$ upper limits (for a single interesting parameter) on their column densities, which are give in Table 1, and shown as dashed lines in Fig. 21. (We note that Si III $\lambda 1206$ in NGC 3783 is buried in the damped Ly $\alpha$ Milky Way absorption.) Acceptable photoionization solutions for the broad absorbing gas in NGC 3783 should have ionization parameters and column densities in the region above the solid lines and below the dashed lines. A solid magenta dot in Fig. 21 shows the photoionization solution used by Mehdipour et al. (2017) for fitting the X-ray spectrum of NGC 3783. Due to the lack of associated X-ray spectral features, this solution is unconstrained in ionization parameter, but it is tightly constrained in total column density, as shown by the bracketing dashed black lines. Thus the combined UV and X-ray data constrain the photoionization state of the obscuring gas to the small, approximately quadrilateral region surrounding the magenta dot in the figure.

\subsection{Variability of the narrow absorption lines}

The intrinsic narrow absorption lines in NGC 3783 are prominent features in its UV spectrum. The proximity and brightness of NGC 3783 has made it a favorite target for trying to understand the physical characteristics and origin of such intrinsic UV absorption features, and their relationship to the blue-shifted $\mathrm{X}$-ray absorption lines comprising the X-ray warm absorber. The extensive Chandra, HST, and FUSE monitoring campaign in 2000-2001 (Kaspi et al. 2002; Gabel et al. 2003a) established the baseline characteristics of these absorbers. There are four discrete velocity components in the UV. We designated them as \#1 through \#4 using the nomenclature established by Gabel et al. (2003a). Adjusting the Gabel et al. (2003a) velocities to the zero point of the more precise redshift of Theureau et al. (1998), we will refer to the four components as \#1 $\left(-1311 \mathrm{~km} \mathrm{~s}^{-1}\right)$, \#2 $\left(-539 \mathrm{~km} \mathrm{~s}^{-1}\right), \# 3\left(-715 \mathrm{~km} \mathrm{~s}^{-1}\right)$, and \#4 $\left(-1018 \mathrm{~km} \mathrm{~s}^{-1}\right)$. Gabel et al. (2005) detected variations in all four components in response to continuum variations as expected for photoionized gas. These variations gave upper limits on the distance of the absorbers in the range $25-50 \mathrm{pc}$; Component \#1 is located more precisely since the density of $\log n_{\mathrm{e}}=4.5 \mathrm{~cm}^{-3}$ determined using metastable $\mathrm{C}_{\text {III }} * \lambda 1176$ places the absorbing gas at $25 \mathrm{pc}$. The 15 year baseline of high-resolution HST spectra of NGC 3783 enables us to examine the behavior of the intrinsic 
Table 1. Properties of the broad absorption troughs in COS observations of NGC 3783.

\begin{tabular}{lcccccccc}
\hline \hline Line & $\begin{array}{c}\lambda_{0} \\
(\AA)\end{array}$ & $\begin{array}{c}v_{1} \\
\left(\mathrm{~km} \mathrm{~s}^{-1}\right)\end{array}$ & $\begin{array}{c}v_{2} \\
\left(\mathrm{~km} \mathrm{~s}^{-1}\right)\end{array}$ & $\begin{array}{c}v_{0} \\
\left(\mathrm{~km} \mathrm{~s}^{-1}\right)\end{array}$ & $\begin{array}{c}\sigma_{v} \\
\left(\mathrm{~km} \mathrm{~s}^{-1}\right)\end{array}$ & $\begin{array}{c}\mathrm{EW} \\
(\AA)\end{array}$ & $\begin{array}{c}\log \left(N_{\text {ion }}\right) \\
\left(\mathrm{cm}^{-2}\right)\end{array}$ & $\begin{array}{c}C_{\mathrm{f}} \\
\text { COS 2016-12-12 } \\
\text { Ly }\end{array}$ \\
\hline N & 1215.67 & -6960 & 0 & -3000 & 830 & $2.16 \pm 0.08$ & $>14.58$ & 0.18 \\
Si II & 1240.51 & -6600 & 0 & -1300 & 1050 & $3.10 \pm 0.11$ & $>15.66$ & 0.37 \\
C II & 1260.42 & -6810 & 0 & - & - & $<0.22$ & $<13.63$ & - \\
Si IV & 1334.53 & -6810 & 0 & - & - & $<0.18$ & $<14.22$ & - \\
C IV & 1398.27 & -7620 & 0 & -3000 & 2300 & $1.33 \pm 0.09$ & $>14.48$ & 0.08 \\
COS 2016-12-21 & 1549.48 & -6810 & 0 & -3000 & 1470 & $6.13 \pm 0.21$ & $>15.51$ & 0.30 \\
Ly $\alpha$ & 1215.67 & -6960 & 0 & -3000 & 860 & $1.25 \pm 0.05$ & $>14.35$ & 0.12 \\
N v & 1240.51 & -6600 & 0 & -1300 & 880 & $2.38 \pm 0.09$ & $>15.56$ & 0.33 \\
Si II & 1260.42 & -6810 & 0 & - & - & $<0.18$ & $<13.68$ & - \\
C II & 1334.53 & -6810 & 0 & - & - & $<0.20$ & $<14.13$ & - \\
Si IV & 1398.27 & -7620 & 0 & -3000 & 1680 & $1.61 \pm 0.10$ & $>14.55$ & 0.11 \\
C IV & 1549.48 & -6810 & 0 & -3000 & 1320 & $4.05 \pm 0.15$ & $>15.31$ & 0.23 \\
\hline
\end{tabular}

Notes. $\lambda_{o}$ is the vacuum rest wavelength of the spectral feature. Absorption troughs span the velocity range given by $v_{1}$ to $v_{2} . v_{0}$ is the transmissionweighted velocity centroid of the trough, $\mathrm{EW}$ is its equivalent width, and $\log \left(N_{\text {ion }}\right)$ is the inferred ionic column density. Assuming the trough is saturated, $C_{\mathrm{f}}$ is the covering factor at the deepest point in the absorption trough.

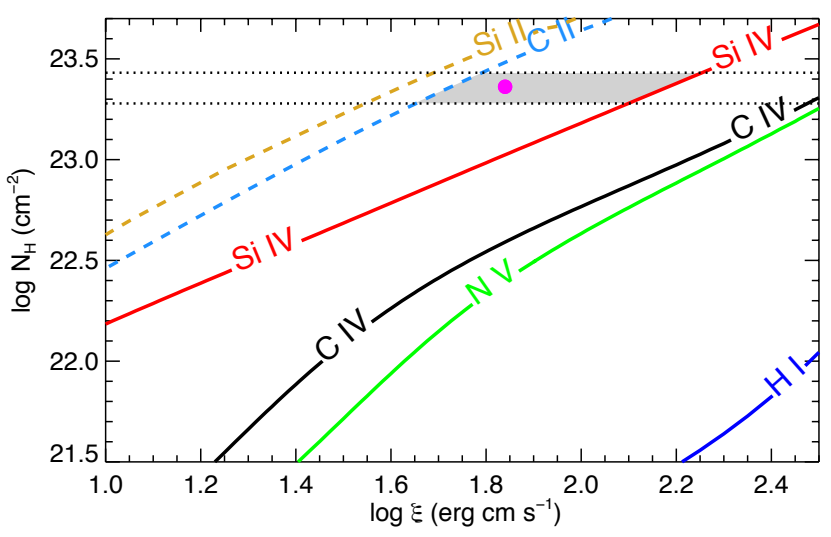

Fig. 21. Constraints on photoionization models for the obscurer in NGC 3783. Dotted black lines give the constraints on total column density allowed by our X-ray spectra in Mehdipour et al. (2017). Solid colored lines specify lower limits on the column densities of the indicated ions. Allowed photoionization solutions lie in the space above these lines. Dashed colored lines give upper limits on the column densities of the indicated low-ionization ions. Allowed photoionization solutions lie in the space below these lines. The shaded parallelogram shows the allowed parameter space, and the magenta dot gives the solution used for fitting the X-ray spectra in Mehdipour et al. (2017).

narrow absorption lines in more detail, and especially in the context of how they have been affected by the obscuration event in 2016.

\subsubsection{Kinematics}

The variability of the narrow absorption lines in NGC 3783 has been both a boon for our understanding of the UV outflow as well as an enigma. In the extensive 2000-2001 monitoring campaign with STIS, the low-ionization portion of Component \#1, designated \#1a by Gabel et al. (2005), showed both absorption in the density-sensitive metastable transitions of $\mathrm{C}$ III* $\lambda 1176$ as well as flux-dependent variability in Si IV that enabled a reliable measurement of the density of the absorbing gas. The puz- zling aspect, however, is that Component \#1 appeared to change in velocity in the sense that it decelerated over the course of the 2000-2001 campaign (Gabel et al. 2003b). Component \#1 appeared to move redward by $90 \mathrm{~km} \mathrm{~s}^{-1}$, from $-1352 \mathrm{~km} \mathrm{~s}^{-1}$ to $-1256 \mathrm{~km} \mathrm{~s}^{-1}$. Scott et al. (2014) showed that this motion appeared to continue in their 2013 spectrum, with Component \#1 moving near to the apparent location of Component \#4 at $-1100 \mathrm{~km} \mathrm{~s}^{-1}$. They note that there is likely motion in other components as well. Component \#3 has disappeared from its original location, and seems to have moved redwards into the region originally occupied by Component \#2, at least to the blue side of the original trough. However, there is no indication that Component \#2 itself has moved redwards.

Our new observations in 2016 plus consideration of the intermediate epoch spectrum in 2011 enables us to take a more comprehensive view. In Figs. 22, 23, and 24 we show normalized spectra at all four epochs $(2001,2011,2013$, and 2016) for Ly $\alpha$, $\mathrm{Nv}$, and Civ. The first thing to notice about the 2016 spectra is that all the absorption features appear to be stronger and deeper. At first this might seem puzzling since the UV continuum in 2016 is brighter than during any of the other epochs. However, this is reminiscent of the behavior of the narrow absorption lines in NGC 5548 during the obscuration event starting in 2014 (Kaastra et al. 2014; Arav et al. 2015; Goad et al. 2016; Mathur et al. 2017; Kriss et al., in prep.). Although the visible UV continuum in NGC 3783 is brighter, the soft X-ray obscuration shows that over $70 \%$ of the soft X-ray continuum is covered by low-ionization, optically-thick gas. This would imply that only a small percentage of the ionizing continuum is illuminating the narrow absorption-line clouds. In fact, with the obscurer allowing only $26 \%$ transmission during the observation on 2016$12-12$, the inferred ionizing UV flux is comparable to the faint state during the 2013 observation.

To illuminate these differences in flux more quantitatively, we compare the UV continuum fluxes at wavelengths both longward and shortward of the Lyman limit at $912 \AA$ in Table 2 . We start with the observed UV continuum fluxes at $1470 \AA$ for all observations, as measured directly from the spectra. From the models we have fit to each spectrum, we extrapolate 


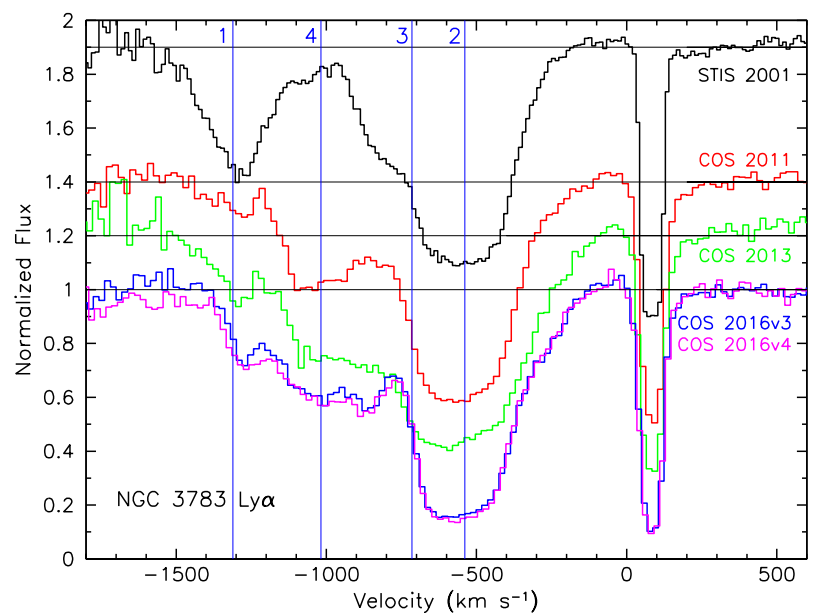

Fig. 22. Normalized HST spectra of the Ly $\alpha$ region from five different epochs as labeled. Velocity is for $\operatorname{Ly} \alpha \lambda 1215.67$ relative to the host galaxy systemic redshift of $z=0.00973$ (Theureau et al. 1998). Fluxes are normalized to range from 0 to 1 . Epochs prior to 2016 are offset vertically by $0.2,0.4$, and 0.9 , with the offset normalizations indicated by thin horizontal black lines. The velocities of Components $1-4$ as given by Gabel et al. (2003b) are marked by thin vertical blue lines and labeled.

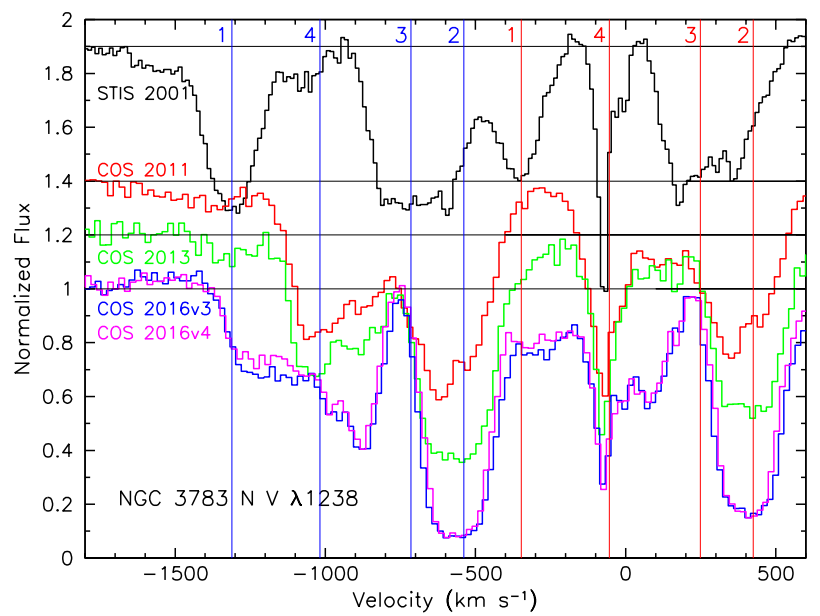

Fig. 23. Normalized HST spectra of the $\mathrm{N} v$ region from five different epochs as labeled. Velocity is for $\mathrm{N} v \lambda 1238.821$ relative to the host galaxy systemic redshift of $z=0.00973$ (Theureau et al. 1998). Fluxes are normalized to range from 0 to 1 . Epochs prior to 2016 are offset vertically by $0.2,0.4$, and 0.9 , with the offset normalizations indicated by thin horizontal black lines. The velocities of Components $1-4$ as given by Gabel et al. (2003b) are marked by thin vertical blue lines and labeled; thin vertical red lines mark the expected locations of the red component of the $\mathrm{N} v$ doublet.

the extinction-corrected power law down to the Lyman limit at $912 \AA$. The column labeled $T_{\mathrm{f}}$ then gives the fraction of light transmitted by the obscurer for each observation, taken from the partial covering models of the X-ray emission in Mehdipour et al. (2017). In the next column we give the extinction-corrected flux at $1470 \AA$. For the last column, we have assumed that the fraction of light transmitted by the obscurer is the same as that measured in the soft X-ray, and then calculate the actual flux at $912 \AA$ after it is blocked by the obscurer. We note that no obscurer was present in 2001, 2011, or 2013.

The consequences of the obscuration in NGC 3783 are most apparent in low-ionization lines such as Si IV (Fig. 25) and C III* $\lambda 1176$ (Fig. 26). Figure 25 compares spectra of the Si IV

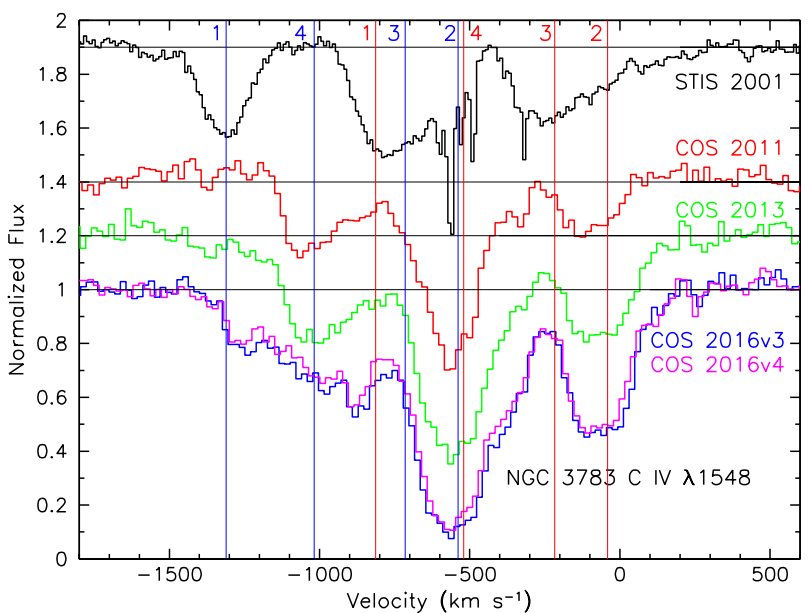

Fig. 24. Normalized HST spectra of the C IV region from five different epochs as labeled. Velocity is for $\mathrm{C}_{\mathrm{IV}} \lambda 1548.195$ relative to the host galaxy systemic redshift of $z=0.00973$ (Theureau et al. 1998). Fluxes are normalized to range from 0 to 1 . Epochs prior to 2016 are offset vertically by $0.2,0.4$, and 0.9 , with the offset normalizations indicated by thin horizontal black lines. The velocities of Components $1-4$ as given by Gabel et al. (2003b) are marked by thin vertical blue lines and labeled; thin vertical red lines mark the expected locations of the red component of the $\mathrm{C}_{\text {IV }}$ doublet.

region at all epochs in calibrated flux units. During the 2001 campaign, Si IV absorption appeared only in Component \#1, most prominently during low-flux states (Gabel et al. 2005). We note that there is no Component \#4 absorption in Si Iv during 2001; in fact, it is only noticeable as a strong feature in $\mathrm{O}$ VI in the FUSE spectrum (Gabel et al. 2003b). Component \#1 shifted in velocity gradually redward during the 2000-2001 campaign (Gabel et al. 2003b), and Scott et al. (2014) suggest that the absorption appearing near the velocity of Component \#4 in 2013 is actually the continued redward evolution in velocity of Component \#1. In the low-flux state of 2013, having "moved" to the location of Component \#4, it appears again. Similarly, Si Iv absorption in Component \#2 only appears in lowflux states. Likewise, $\mathrm{C}_{\text {III }}{ }^{*} \lambda 1176$ absorption was only associated with Component \#1 in the 2000-2001 campaign, and was strongest in the low states (Gabel et al. 2005). As Fig. 26 shows, $\mathrm{C}_{\text {III }} * \lambda 1176$ has reappeared during the 2016 obscuration observations as a shallow depression near the original velocity of Component \#4, but more likely representing the evolution in velocity of gas associated with Component \#1. As Table 2 shows, the ionizing flux at $912 \AA$ in 2016 is nearly as low as in 2013, if most of the intrinsic continuum is hidden by the obscurer. This shadowing of the narrow-absorption-line gas by the obscurer then explains the appearance of absorption associated with Components \#1 and \#2 during the obscuration event in 2016.

\subsubsection{Photoionization response}

Several factors complicate the interpretation of the absorptionline profiles in NGC 3783. As Gabel et al. (2003a) showed in their analysis of the full Lyman series using both the STIS and FUSE spectra, Ly $\alpha$ is heavily saturated. The absorption lines are broad and blended. In C IV, the velocity spacings lead to overlap between the blue and red lines of the $\mathrm{C}$ IV doublet for Components \#1 and \#3, and for \#2 and \#4. In $\mathrm{Nv}$ the individual troughs are only slightly blended, but the red transition of Component \#4 is contaminated by foreground interstellar 
Table 2. UV continuum fluxes in NGC 3783.

\begin{tabular}{lccccc}
\hline \hline Observation & $F(1470 \AA)^{a}$ & $F(912 \AA)^{b}$ & $T_{\mathrm{f}}{ }^{c}$ & $\begin{array}{c}F(1470 \AA)^{d} \\
\text { (inferred) }\end{array}$ & $\begin{array}{c}F(912 \AA)^{e} \\
\text { (inferred) }\end{array}$ \\
\hline STIS 2001 & 3.76 & 16.4 & 1.00 & 8.47 & 16.4 \\
COS 2011 & 3.52 & 13.3 & 1.00 & 7.98 & 13.3 \\
COS 2013 & 1.53 & 5.9 & 1.00 & 3.51 & 5.9 \\
COS 2016-12-12 & 7.24 & 27.1 & 0.26 & 16.6 & 7.0 \\
COS 2016-12-21 & 7.97 & 30.2 & 0.32 & 18.6 & 9.7 \\
\hline
\end{tabular}

Notes. ${ }^{(a)}$ Observed continuum flux at $1470 \AA\left(10^{-14} \mathrm{erg} \mathrm{cm}^{-2} \mathrm{~s}^{-1} \AA\right)$. ${ }^{(b)}$ Fitted continuum flux extrapolated to $912 \AA$ and corrected for extinction assuming $E(B-V)=0.107\left(10^{-14} \mathrm{erg} \mathrm{cm}^{-2} \mathrm{~s}^{-1} \AA\right) .{ }^{(c)}$ Fraction of the continuum transmitted by the obscurer (Mehdipour et al. 2017). ${ }^{\left({ }^{(d)}\right.}$ Extinctioncorrected continuum flux at $1470 \AA\left(10^{-14} \mathrm{erg} \mathrm{cm}^{-2} \mathrm{~s}^{-1} \AA\right)$. ${ }^{(e)}$ Inferred ionizing continuum flux at $912 \AA$ corrected for extinction and diminished by transmission through the obscurer $\left(10^{-14} \mathrm{erg} \mathrm{cm}^{-2} \mathrm{~s}^{-1} \AA\right)$.

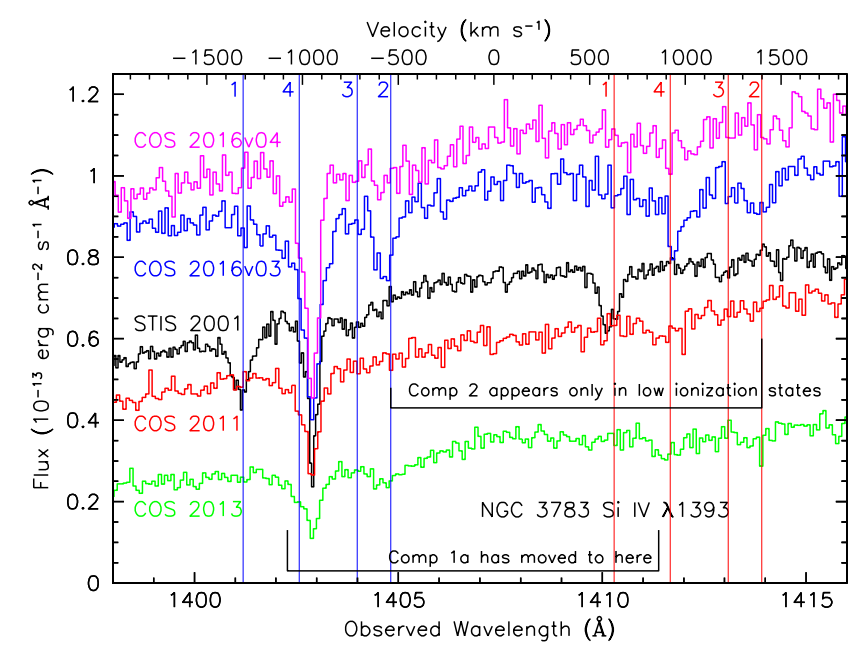

Fig. 25. HST spectra of the Si Iv region from five different epochs as labeled. Fluxes and wavelengths are as observed. The wavelengths of Components 1-4 as given by Gabel et al. (2003b) are marked by thin vertical blue lines and labeled; thin vertical red lines mark the expected locations of the red component of the Si iv doublet. Note that absorption in Component \#2, which is strong only in low-flux states in all prior observations, appears strongest during the 2016-12-12 COS observation, which was taken at the time that X-ray obscuration was strongest. Also, the low-ionization Component \#1a, which is the "decelerating" absorption-line cloud (Gabel et al. 2003b; Scott et al. 2014), has decelerated to an observed wavelength of $1403 \AA$, where it is blended with Galactic Si Iv $\lambda 1403$.

absorption by $\mathrm{S}$ II $\lambda 1250$. Since $\mathrm{N} v$ has the cleanest profiles, we use it as a starting point for all of our analysis. In the threeyear interval from 2013 to 2016, we note that the figures show little additional apparent motion of Component $\# 1$. In fact, its motion appears to have halted by the time the 2011 spectrum was recorded where its location differs little from 2013 or 2016. Another intriguing aspect of our 2016 spectra is that absorption near the original position of Component \#1 has re-appeared.

Absorption at the original locations of Components \#2 and \#4 show a clear ionization response, perhaps indicating that these are stable, persistent features. Absorption at the velocity of Component \#4 only appears in the low flux state of the COS 2013 observation. Initially it may seem surprising that it is also present in the first observation on 2016-12-12 (and not strongly in the second), but this can be understood as another example of the line-of-sight shadowing by the transient soft-X-ray obscuration, as for the NGC 5548 obscurer (Arav et al. 2015). If the UV flux is scaled down by the transmission of the obscurer (only $26 \%$ for

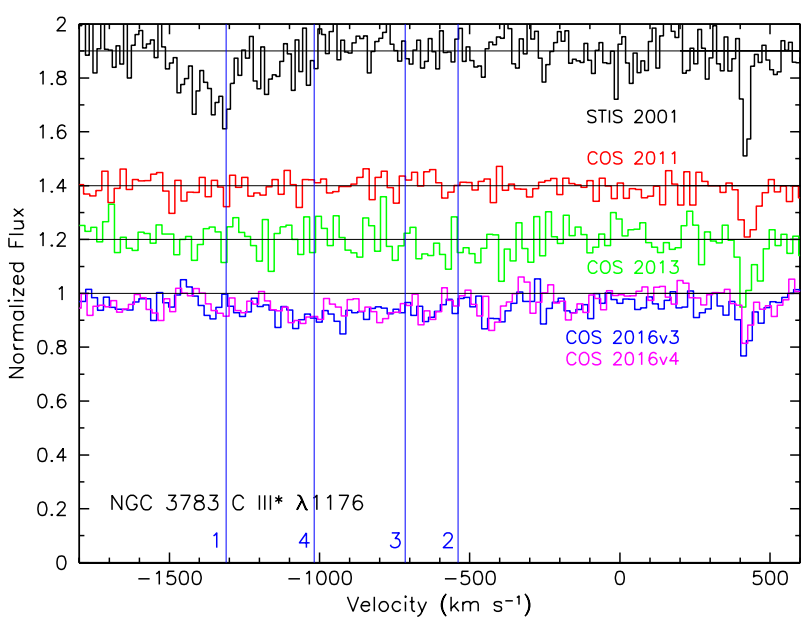

Fig. 26. Normalized HST spectra of the $\mathrm{C}_{\mathrm{III}} * \lambda 1176$ region from five different epochs as labeled. Velocity is for the strongest $\mathrm{C}_{\mathrm{III}}{ }^{*} J=2$ transition at $1175.71 \AA$ relative to the host galaxy systemic redshift of $z=0.00973$ (Theureau et al. 1998). Fluxes are normalized to range from 0 to 1 . Epochs prior to 2016 are offset vertically by $0.2,0.4$, and 0.9 , with the offset normalizations indicated by thin horizontal black lines. The velocities of Components 1-4 as given by Gabel et al. (2003a) are marked by thin vertical blue lines and labeled.

the 2016-12-12 observation), the inferred ionizing flux is actually lower than in 2013. Similarly, in the 2016-12-21 observation, the obscuration is not as opaque, the intrinsic continuum is slightly brighter, and the inferred ionizing flux is higher than for the COS 2013 observation.

Similar arguments apply to the depth of the red side of the absorption trough in Component \#2, although the interpretation is slightly more complicated due to the different covering fractions of the line and continuum for this feature, and the strongly varying intensity of the emission lines relative to the continuum among the HST observations of NGC 3783. Based on the analysis of the Lyman lines, Gabel et al. (2003a) show that the narrow absorption features have different covering factors for the lines and the continuum. The depth of the troughs in Ly $\alpha$, which is certainly saturated, for the 2016 observation show that over $92 \%$ of both line and continuum is covered.

To try to unravel the behavior of the narrow absorption components, we also have examined their changing structure in the context of potential responses to changes in the ionizing flux illuminating the absorbing clouds or filaments. We start with the structures at the highest blue-shifted velocities, Component \#1, then Components \#4, \#3, and \#2. 
Component \#1. This component was strong and well defined in 2001 (and earlier). As described by Gabel et al. (2005), it appears to have both a low-ionization component, \#1a, that appears in all ionic species, including $\mathrm{Si}$ IV and $\mathrm{C}$ III*. It is blended with a high-ionization portion called $1 \mathrm{~b}$, which is needed to explain the $\mathrm{N} v$ and $\mathrm{O}$ vi strengths. It is the low-ionization portion, \#1a, that appears to decelerate (Gabel et al. 2003b), although the shift in the line centroid is seen in all ions. The most definitive detection is in our 2016-12-12 observation (v3), where it appears in both $\mathrm{Si}$ IV and $\mathrm{C}$ III* The $\mathrm{C}$ III* absorption associated with Component \#1 in 2016 appears as a broad, shallow depression at the velocity of Component \#4, visible in Fig. 26.

Similarly, the Ly $\alpha$ absorption formerly associated with Component \#1 in 2001 has also shifted to the position of Component \#4 in the 2011, 2013, and 2016 spectra. This new location for \#1a also shows an apparent response to ionizing flux in that it is deepest for the 2013 spectrum, followed by 2016, and then 2011 . This is consistent with higher neutral hydrogen column densities during observations with lower ionizing flux (i.e., observed UV flux corrected by transmission of the obscurer).

This apparent motion in Component \#1a seems to have separated it kinematically from the high-ionization Component \#1b. Just redward of the 2001 velocity of Component \#1, a new component appears in 2011 in Ly $\alpha$ at $-1280 \mathrm{~km} \mathrm{~s}^{-1}$. We suggest that this is the counterpart to high-ionization Component \#1b. It also shows an apparent response to changes in the ionizing flux, being deepest in 2013 and 2016, and shallower in 2011. Note that this velocity is also more consistent with the blue side of the $\mathrm{X}$ ray absorption troughs in the high ionization ions, for example, Fig. 9 of Scott et al. (2014), comparing profiles for Mg XI and $\mathrm{Mg}$ XII to the UV ion $\mathrm{N}$.

The behavior of this feature in $\mathrm{Nv}$ and $\mathrm{C}$ IV corroborates its identification as the high-ionization Component \#1b. The feature stands out as well defined in N v and C IV in spectra from 2016 when the ionizing continuum was obscured, but appears only weakly in the prior spectra. As shown by the photoionization models in Fig. 7 of Gabel et al. (2005), this is consistent with it having an ionization parameter $\log U>-1.5(\log \xi>0.0)$, lying well beyond peak ionization for $\mathrm{Nv}$ and $\mathrm{C}$ IV during 2001 and 2011, but accumulating higher column densities in those ions at the lower ionization parameters more likely present during the obscured state.

Component \#4. As Scott et al. (2014) note, the original Component \#1 appears to have moved to the velocity of Component \#4. In $\mathrm{N} v$ this transition in velocity happened by the time of the 2011 observation. There is no discernible change in velocity between 2011 and 2013, but the feature is much weaker in 2016 .

In addition to this possible motion of Component \#1a, another trough appeared redward of the Component \#4 location at $-880 \mathrm{~km} \mathrm{~s}^{-1}$ in 2011 , and this feature persists in 2013 and 2016. Its depth has no relation to the strength of the ionizing flux - it is weakest in 2013 when the ionizing flux was weakest, stronger during the bright 2011 epoch, and at its strongest during the 2016 obscuration event. However, in Ly $\alpha$ this feature shows a more consistent response to changes in the ionizing flux level. It is strongest in 2013 when the ionizing flux level was lowest, weakest in 2011 when it was highest, and in between during the epoch of obscuration in 2016.

Component \#3. Component \#3 essentially disappears after 2001. Scott et al. (2014) note its absence in 2013, but it is also not present in the 2011 or the 2016 spectra.

Component \#2. Component \#2 was the most prominent absorber in Ly $\alpha$ in 2001, but it was not exceptionally strong in any other ion. Starting in 2011, it develops a profile in $\mathrm{N} \mathrm{v}$ and $\mathrm{C}$ IV more similar to its appearance in Ly $\alpha$. Variations in Component \#2 are analyzed most cleanly in the $\mathrm{Nv}$ transitions, where it is unblended. It is saturated at all epochs in Ly $\alpha$, and it is only visible in low-ionization epochs in Si IV (2013 and 2016). In C IV, the blue component overlaps the red trough of Component \#4, or the decelerated red trough of Component \#1a. The red trough of Component \#2 is unblended. It therefore can be used to corroborate inferences derived from analysis of the $\mathrm{N} v$ doublet, but it cannot provide an independent measure of covering fraction and optical depth.

The transition in the morphology of the Component \#2 absorption profile from 2001 to 2011 is suggestive of (1) Component \#3 having decelerated from its position in 2001 to form the blue side of the Component \#2 trough, and (2) the red half of the trough corresponding to the original 2001 location of Component \#2. The evolution in strength of Component \#2 is consistent with a response to changes in the ionizing flux. In 2001, when the ionizing UV flux was strongest, Component \#2 was at its weakest. During the obscured epoch of 2016, when the ionizing UV is weakest, Component \#2 has its deepest troughs, with the $\mathrm{N} \mathrm{v}$ profile having an appearance similar to the saturated Ly $\alpha$ profile. The intermediate depths in 2011 and 2013 are in proportion to the relative strengths of the UV flux at those epochs, both of which were fainter than 2001. The red transition of Component \#2 in C IV shows this same pattern of changes in trough depth. Since Ly $\alpha$ is strongly saturated, this suggests that this component is highly ionized, as shown in Fig. 7 of Gabel et al. (2005), with the C IV and $\mathrm{N} v$ ionization fractions being well past their peaks.

\section{Discussion}

Our simultaneous UV spectra of NGC 3783 quantify the broad, fast absorption that appeared in the blue wings of the highionization UV resonance lines at the same time as the appearance of strong soft X-ray obscuration (Mehdipour et al. 2017). Our detailed analysis of the emission-line profiles show that broad absorption on the blue wings of the permitted emission lines (and the absence of absorption in excited-state lines such as $\mathrm{He}$ II $\lambda 1640$ and $\mathrm{H} \beta$ ) provides a more physically consistent description than an arbitrary set of emission components. Combining the spectral diagnostics of the obscurer in our UV observations with the total column density measured with the X-ray spectra enables us to determine the ionization state $(\log \xi=$ $1.84_{-0.2}^{+0.4} \mathrm{erg} \mathrm{cm} \mathrm{s}^{-1}$ ) and kinematics of the outflowing gas responsible for the obscuration. Indeed, without the UV observations, it would not even have been possible to assert that the soft Xray obscuration was due to an outflow since there are no prominent spectral features in the heavily absorbed X-ray spectrum. Our UV spectra show that the absorption extends from near zero velocity to a maximum of $\sim-6200 \mathrm{~km} \mathrm{~s}^{-1}$, with a flux-weighted mean of $-2840 \mathrm{~km} \mathrm{~s}^{-1}$.

As in NGC 5548 (Kaastra et al. 2014), the strength and depth of the UV absorption varies in concert with variations in the X-ray obscuration. When the obscuration is strongest, on 2016-12-12, the UV absorption is strongest. As the obscuration lessened, as shown in the 2016-12-21 observation with XMM-Newton, the UV absorption also diminished. This could be seen not only in the depths of the broad absorption features, but also in its inferred influence on the ionizing UV continuum. Low-ionization features in the intrinsic narrow absorption lines of NGC 3783 such as C III* $\lambda 1176$ and Si Iv $\lambda \lambda 1393,1402$ become deeper when the X-ray obscuration is strongest. As in NGC 5548 (Arav et al. 2015), this argues that the X-ray and UV 
obscurer lies interior to the clouds producing the intrinsic narrow absorption lines.

Nearly two decades of high spectral resolution observations of the narrow absorption-line features in NGC 3783 show that they are not as kinematically stable as other AGN we have studied intensively, for example, Mrk 509 (Kriss et al. 2011; Arav et al. 2012), or NGC 5548 (Arav et al. 2015). The variations in the narrow absorption lines in NGC 3783 discussed in Sect. 3.4 show that the behavior of these absorption features is not as simple as the stable absorption troughs seen in NGC 5548. In NGC 5548, these troughs maintain their velocities; they show variations consistent with constant column density and covering factor and simple changes in ionic column density determined purely by variations in the ionizing flux. In NGC 3783, even for features that seem to have been stable in velocity from 2011 to 2016, we see changes in both ionic column density and in covering factor. Although some columndensity changes are correlated with changes in ionizing flux (e.g., Component \#1a and Component \#2), others are not (Component \#1b, Component \#3, and Component \#4). Thus, we must be viewing configurations of absorbing clouds that are clumpy, and possibly changing their arrangement along the line of sight. Given the relative stability in velocity of some of the features, it seems that rather than having clouds or filaments crossing our line of sight, or decelerating, that an alternative possibility might be that the multiple velocities we see are associated with dense clouds or filaments that are moving at fixed velocity. These dense entities are a source of material, and, in and of themselves, they have low enough covering fraction so as not to cause noticeable absorption. The absorption we see is then caused by material ablated or evaporated from the outer portions of these dense sources. This material is photoionized, heats up, expands, and thus varies in covering fraction and ionization, giving rise to the variable features we see. Dense knots like this, which form and evaporate can form naturally as thermal instabilities in a radiatively accelerated flow (Proga \& Waters 2015).

Rather than invoking deceleration to explain the changing kinematics of these clumps, transverse motions of clouds along our line of sight seems more plausible. These transverse motions may be related to velocity shear in outflowing streamlines that have both radial and toroidal motions defining their outflow trajectories. Based on the measured electron density and column density of Component \#1b, Gabel et al. (2005) infer a transverse size of $10^{16} \mathrm{~cm}$ for a uniform cloud. At the distance of $30 \mathrm{pc}$ based on the photoionization solution, Keplerian velocities at this location would be of order $200 \mathrm{~km} \mathrm{~s}^{-1}$, which would include the gravitational potential of the host galaxy as well as the black hole. Thus it would take 2.1 years for the cloud to traverse our line of sight. Since we see dramatic changes in ten years or less, if these were caused by transverse motion, the cloud would have to be elongated along our line of sight by a factor of ten relative to its width. If "clouds" are really filaments, perhaps organized along streamlines or magnetic field lines (e.g., Fukumura et al. 2010), this is not implausible.

\subsection{Structure and evolution of the broad line region}

Based on the X-ray partial covering of the obscurer, which suggests its transverse size is comparable to the size of the $\mathrm{X}$-ray emitting continuum region, and the variability timescale of $\sim 1$ day in the X-ray, Mehdipour et al. (2017) infer a density of $\sim 2.6 \times 10^{9} \mathrm{~cm}^{-3}$ and an approximate location of $\sim 10$ lt-days from the source. The location, density, and the kinematics of

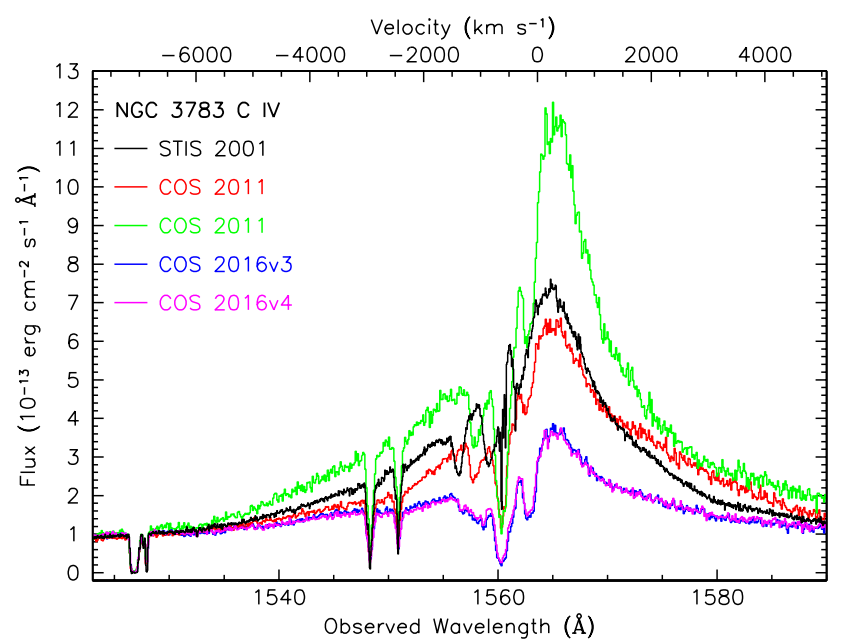

Fig. 27. Comparison of the $\mathrm{C}$ IV emission line profiles for NGC 3783 in the observations with STIS 2001 (black), COS 2011 (red), COS 2013 (green), and COS 2016 Visit 3 (blue) and COS 2016 Visit 4 (magenta). The profiles have all been scaled to the flux level of COS 2016 Visit 4 at $1525 \AA$.

the obscuring outflow all suggest that it may be associated with the broad line region in NGC 3783. As we showed in Sect. 3, the broad emission line profiles have also changed substantially between 2001 and 2016.

The extensive reverberation mapping campaign conducted on NGC 3783 in 1992 by the International AGN Watch (Reichert et al. 1994; Stirpe et al. 1994; Onken \& Peterson 2002) showed that the UV emission lines respond to variations in the continuum flux as expected if they are reprocessing the ionizing radiation from the central source. Refined analysis of these data (Onken \& Peterson 2002) determine a mean lag for the C IV emission line of 3.8 days. During the 1992 campaign, the line fluxes tracked the continuum closely, with no dramatic changes in the line profile. However, as demonstrated in Sect. 3, the broad emission line profiles observed by COS in 2016 differ dramatically from those seen with STIS in 2001. The archival COS observations from 2011 and 2013 enables us to study the evolution of these profiles. We have fit both of these spectra using the same procedures as for the unobscured STIS spectrum. The best-fit parameters for each are tabulated in Table A.4. To show a more comprehensive history of these variations, we compare the $\mathrm{C}$ IV emission line profiles for all four epochs from 2001 to 2016 in Fig. 27. The most striking aspect of this comparison is the large variation in equivalent width among these observations. Table 3 summarizes the total fluxes and equivalent widths (EW) of the C IV emission lines at all four epochs in our study. We note that although both the continuum and the $\mathrm{C}$ IV flux were at a maximum in 2016, the equivalent width was at a minimum. Conversely, when flux was at a minimum in 2013, the equivalent width was at a maximum. The narrow core of the line dominates the emission profile (at velocities $<500 \mathrm{~km} \mathrm{~s}^{-1}$ ) in 2013 , but it makes only a minor contribution in 2016. In fact, the narrow component stays relatively constant in flux, similar to the narrow core in NGC 5548 (Crenshaw et al. 2009).

Figure 28 shows these variations quantitatively. The very broad component varies roughly in proportion to the variations in the continuum flux. The medium-broad (FWHM $2838 \mathrm{~km} \mathrm{~s}^{-1}$ ) component shows the most variation, virtually disappearing during the COS observations of 2016. This same kinematic component is also much weaker in the $\mathrm{Nv}, \mathrm{Si}$ Iv, 


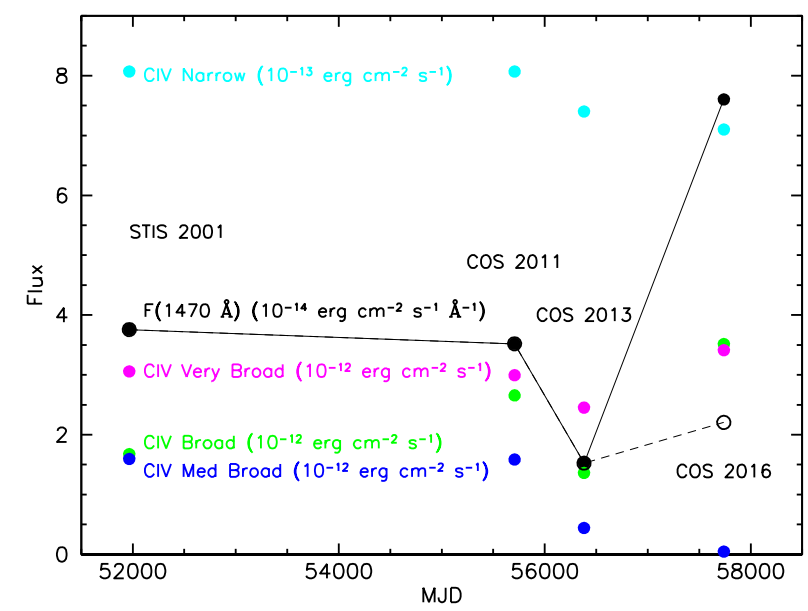

Fig. 28. Fluxes at the epochs of the STIS 2001, COS 2011, COS 2013 , and COS 2016 observations of NGC 3783 in the continuum at $1470 \AA$ (black) and in the several components of the $\mathrm{C}_{\mathrm{IV}}$ emission line: the narrow component (cyan), the medium-broad component (green), the broad component (blue), and the very broad component (magenta). Fluxes are scaled as noted in the labels in the figure. The continuum points are connected by a thin black line. The open circle in the 2016 line shows the value of the continuum flux if we scale it down by the transmission of the obscurer at the epoch of the COS observations.

Table 3. C Iv Emission-line fluxes and equivalent widths in NGC 3783.

\begin{tabular}{lccc}
\hline \hline Observation & $F(1470 \AA)^{a}$ & C Iv Flux $^{b}$ & C IV EW $^{c}$ \\
\hline STIS 2001 & 3.76 & 718 & 204 \\
COS 2011 & 3.52 & 676 & 200 \\
COS 2013 & 1.53 & 504 & 343 \\
COS 2016 (average) & 7.67 & 777 & 104 \\
\hline
\end{tabular}

Notes. ${ }^{(a)}$ Observed continuum flux at $1470 \AA\left(10^{-14} \mathrm{erg} \mathrm{cm}^{-2} \mathrm{~s}^{-1} \AA\right)$. ${ }^{(b)}$ Total observed $\mathrm{C}_{\mathrm{IV}}$ emission-line flux $\left(10^{-14} \mathrm{erg} \mathrm{cm}^{-2} \mathrm{~s}^{-1}\right) .{ }^{\left({ }^{(c)}\right.} \mathrm{C}$ IV emission-line equivalent width (EW) $(\AA)$.

He II, and $\mathrm{H} \beta$ line profiles ${ }^{3}$. Although our decomposition of the line profile is not unique, given the kinematic relationships between line width and reverberation lags established in many reverberation campaigns, we can roughly decompose the $\mathrm{C}$ IV emission-line profile into a correspondence between width and distance from the central source using the corresponding Keplerian orbital velocities in the potential of the $2.35 \times 10^{7} M_{\odot}$ black hole (Bentz \& Katz 2015) in NGC 3783. The Keplerian velocity corresponding to the mean lag of 3.81 t-days is $5630 \mathrm{~km} \mathrm{~s}^{-1}$. For our model of the C IV profile, the narrow core $\left(937 \mathrm{~km} \mathrm{~s}^{-1}\right)$ lies at a distance of 137 lt-days, the medium-broad component $\left(2838 \mathrm{~km} \mathrm{~s}^{-1}\right)$ at $15 \mathrm{lt}$-days, the broad component $\left(4576 \mathrm{~km} \mathrm{~s}^{-1}\right)$ at 5.8 lt-days, and the very broad component $\left(10030 \mathrm{~km} \mathrm{~s}^{-1}\right)$ at 1.2 lt-days.

In the context of our model of the line profile, both 2013 and 2016 represent unusual states for the broad line region of NGC 3783. Overall, the continuum in 2013 is $2.5-5 \times$ fainter than in 2001 or 2016 , yet the very broad component of the $C_{\text {IV }}$ emission-line flux is dimmer by only $20-30 \%$. If the BLR is reprocessing continuum radiation, to compensate for the reduced

\footnotetext{
The medium-broad component of $\operatorname{Ly} \alpha$ does not share this behavior. Strong absorption due to damped Milky Way Ly $\alpha$ absorption blends with all components on the blue wing of Ly $\alpha$ in NGC 3783 and adds considerable degeneracy to our decomposition of the line profile.
}

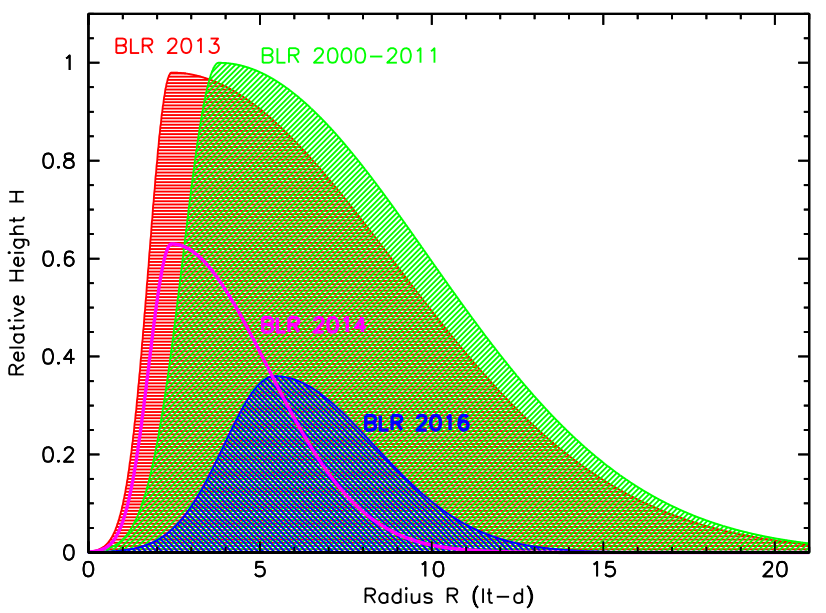

Fig. 29. Schematic representation of the evolution of the BLR in NGC 3783. The green shaded area represents the vertical structure and radial extent of the BLR during the period from 2000 to 2011 . The red shaded area shows the inferred structure in 2013, when the continuum flux was $2.5 \times$ less, but the EW of C Iv was $70 \%$ higher. The magenta curve is the hypothetical structure in 2014, when the continuum flux was still low after the BLR had dynamically adjusted to the lower flux level. The blue shaded area is the inferred structure at the time of the obscuration event in 2016.

continuum flux and still radiate nearly as profusely, the covering factor of the very broad portion of the $\mathrm{C}$ IV profile must be higher. This implies that in the faint state of 2013, the inner part of the BLR must have puffed up vertically. At the same time, we note that the medium-broad component at $2838 \mathrm{~km} \mathrm{~s}^{-1}$ virtually disappeared in 2016.

We can interpret these changes in the context of recent models in which a dust- and line-driven wind from the accretion disk create the BLR (Czerny \& Hryniewicz 2011; Czerny et al. 2017; Baskin \& Laor 2018). Although these models do not yet quantitatively predict sizes and velocities for the BLR that match the emission-line profiles and velocities observed in typical AGN (Czerny et al. 2017), they offer a physical framework that may explain some of the qualitative changes we observed in the spectra of NGC 3783 over the years. We hypothesize that the dramatic drop in continuum flux in 2013 (or shortly before) initiated a reconfiguration of the BLR in NGC 3783. Figure 29 illustrates this evolution schematically.

From the 1992 reverberation-mapping campaign through the STIS observations in 2000-2001 and the COS observation in 2011, the broad-line profiles in NGC 3783 remained similar in shape and equivalent width. This apparently stable configuration is represented by the shaded green area in Fig. 29. The shape is chosen to resemble line emissivity distributions typical of reconstructions from reverberation-mapping results (Krolik et al. 1991; Grier et al. 2013). The peak is set at the $\mathrm{C}$ IV mean lag of 3.8 lt-days. To scale this configuration to other epochs in the history of NGC 3783 observations, we use the scaling relations of Baskin \& Laor (2018) for a dusty wind-driven BLR. The peak radius of the broad line region, $R_{\mathrm{BLR}}$, scales with luminosity, $L$, as $R_{\mathrm{BLR}} \sim L^{1 / 2}$. The height of the broad line region, $H$, also scales with luminosity as $H \sim L^{1 / 2}$. Thus, when the continuum brightness drops in 2013, the peak of the BLR emissivity shifts to a lower radius, $R=2.4$ lt-days. Of course, the BLR can not change its shape instantaneously. For the radiation-pressure driven winds in the Czerny et al. (2017) and Baskin \& Laor (2018) models, the relevant timescale is the local dynamical timescale for material moving up or down from 
the accretion disk, set by the vertical component of the gravitational force exerted by the central black hole. The free-fall time for material falling back to the disk after the radiation pressure disappears is

$t_{\mathrm{ff}}=\left(\frac{2 R^{3}}{G M_{\mathrm{BH}}}\right)^{1 / 2}$.

So, in 2013, the location of peak emissivity is expected to move inward to 2.4 lt-days in response to the reduced brightness of the central source and the accretion disk. The expected BLR height at that location would also be expected to be lower, at $\sim 64 \%$ of the peak height in 2000-2011. However, it is likely that highly ionized gas above the disk would drop in ionization very quickly, producing BLR-like emissivity at large heights. The red shaded area in Fig. 29 reflects the smaller $R_{\mathrm{BLR}}$, but a height consistent with the higher observed equivalent width of the C IV emission line. Gas at these heights either at small radii in the disk or at larger radii can not be supported via radiation pressure, but the dynamical adjustments in the vertical extent of the BLR would take months to years to adjust to this lower luminosity. At 2.4 lt-days, the timescale is $t_{\mathrm{ff}}=0.3$ years; at $10 \mathrm{lt}$-days, the peak radius for $\mathrm{H} \beta$ in the 1992 reverberation campaign, it is $3.4 \mathrm{yr}$. The red shaded area in Fig. 29 reflects these delays. We do not know when the drop in continuum flux actually occurred prior to the 2013 COS observation, but the large equivalent width observed in the emission lines implies that gas at those innermost radii has not yet had time to fall back toward the disk. Therefore we infer that the drop in flux happened very soon before the 2013 observation. Similarly, since the gas at larger radii would take years to fall back, the red shaded area shows a similar height to the prior shape represented by the green shaded area.

We have no observations of NGC 3783 in 2014, so the magenta curve in Fig. 29 is a hypothetical view of how the BLR would have responded dynamically to the lower flux observed in 2013, had it remained at that low flux level. By then, gas at heights supported by the normal flux state in 2000-2011 would have had time to fall back toward the accretion disk. The peak height of the BLR is adjusted by the square root of the flux ratios between 2001 and 2013, and the vertical extent of the BLR at all radii drops significantly due to the loss of radiation-pressure support.

Swift observations of NGC 3783 (Kaastra et al. 2018) show that NGC 3783 was still in a low flux state in mid-to-late 2016, several months before the obscuration event in late 2016 (Mehdipour et al. 2017). Therefore, even though NGC 3783 had brightened dramatically, gas in the BLR would not have had time to move in response to these changes. The blue shaded area in Fig. 29 shows the peak of the BLR emissivity moving to a larger radius, 5.4 lt-days, but not changing much in vertical height since it has not yet had time to respond dynamically to the increased radiation pressure. The height shown in Fig. 29 is scaled to give the observed ratio of $C$ IV equivalent width in 2016 to that in 2000-2001. This height, however, is about a factor of four lower than the increased flux in 2016 could potentially support via radiation pressure. Thus, gas at all radii is likely flowing upward and outward from the plane of the accretion disk as it is accelerated by the increased radiative flux. In addition, there is a substantial amount of gas at radii interior to 5 lt-days at high elevations that is now exposed to a much stronger ionizing flux. We suggest that this gas and the outflow induced by the increased ionizing flux is the source of the obscuring outflow observed by Mehdipour et al. (2017).

The changes we have observed in the structure of the BLR in NGC 3783, and perhaps in the mechanisms driving these changes, are similar to the more extreme variability seen in "changing-look" AGN. These AGN at their most extreme appear to change from Type 1, with bright, typical BLRs, to Type 2, with almost no remaining observational trace of the BLR. Examples date back to the earliest studies of AGN (e.g., Mrk 6; Khachikian \& Weedman 1971) and NGC 4151 (Penston \& Perez 1984). More recent, intensively studied examples include Mrk 590 (Denney et al. 2014; Mathur et al. 2018) and HE1136-2304 (Zetzl et al. 2018). In such objects, the continuum luminosity varies by one to two orders of magnitude; in the low-luminosity states, the BLR can nearly completely disappear, the extreme version of the dimunition we observed in NGC 3783. In models of the BLR produced by disk-driven winds, the BLR is expected to completely disappear below a critical luminosity required to sustain the outflow (Elitzur \& Ho 2009; Elitzur et al. 2014), producing an AGN that is a "true Type 2", where the BLR is not merely obscured, but completely absent. Elitzur \& Ho (2009) cite a threshold for such a transition at a bolometric luminosity of $L_{\mathrm{bol}}=1.5 \times 10^{38}\left(M_{\mathrm{BH}} / 10^{7} M_{\odot}\right)^{2 / 3} \mathrm{erg} \mathrm{s}^{-1}$. For the black hole mass of $2.35 \times 10^{7} M_{\odot}$ in NGC 3783 , this threshold is far below the bolometric luminosity of $2.8 \times 10^{44} \mathrm{erg} \mathrm{s}^{-1}$ (based on the spectral energy distributions of Mehdipour et al. 2017), so we would not expect such a drastic reconfiguration of the BLR.

Comprehensive surveys of candidates in the Sloan Digital Sky Survey (SDSS) reveal that 30-50\% of quasars exhibit extreme variability characteristic of changing-look AGN that lead to dramatic changes in the BLR (MacLeod et al. 2016, 2018; Rumbaugh et al. 2018). These objects have systematically lower Eddington ratios $(<10 \%)$, and are similar in luminosity and Eddington ratio to Seyfert 1 s like NGC $3783\left(L_{\text {bol }} / L_{\text {Edd }} \sim\right.$ 0.02). Thus, detailed study of a less extreme event such as we have observed may offer some insights into the physics governing AGN classification in general. A crucial difference between the events in NGC 3783 and in the changing-look quasars is the appearance of heavy obscuration accompanying the return to normal luminosities in NGC 3783. This may imply that in changing-look objects that much of the material in the accretion disk and the accompanying gas that supplies the wind for the BLR may have been emptied from the system during the low-luminosity state. This would leave no excess material to be ejected as the obscuring outflow during the return to normal brightness in the changing-look AGN.

\subsection{Impact on the host galaxy}

A high velocity wind originating in or near the BLR in an AGN can potentially have a significant impact on the host galaxy since the kinetic luminosity of an outflow varies with velocity, $v$, as $v^{3}$. For a spherical shell moving at this velocity with a radius $R$, a total hydrogen column density $N_{\mathrm{H}}$ and covering fraction $\Delta \Omega$, the mass flux and kinetic luminosity of the outflow are

$\dot{M}=4 \pi \Delta \Omega R N_{\mathrm{H}} \mu m_{\mathrm{p}} v$

$\dot{E}_{\mathrm{k}}=\frac{1}{2} \dot{M} v^{2}$

where $m_{\mathrm{p}}$ is the proton mass and $\mu=1.4$ is the mean molecular weight. The properties of the obscurer in NGC 3783 suggest it might have sufficient kinetic luminosity to have an evolutionary impact on the host galaxy.

Mehdipour et al. (2017) derive a radial distance for the obscurer of 10 lt-days; the $\mathrm{C}$ IV emission line region has a reverberation radius of $3.8 \mathrm{lt}$-days (Reichert et al. 1994). For a central black hole mass of $M_{\mathrm{BH}}=2.35 \times 10^{7} M_{\odot}($ Bentz \& Katz 2015), 
Keplerian velocities at 10 lt-days and 3.8 lt-days are $3500 \mathrm{~km} \mathrm{~s}^{-1}$ and $5600 \mathrm{~km} \mathrm{~s}^{-1}$, respectively. These are typical of the velocities of the deepest point in the UV absorption troughs of the obscurer $\left(-3000 \mathrm{~km} \mathrm{~s}^{-1}\right)$, and their highest velocity extent from line center at $-6200 \mathrm{~km} \mathrm{~s}^{-1}$, which suggest that the kinematics of the obscurer are similar to those of the BLR. Assuming a covering fraction of $\Delta \Omega=0.25$, these velocities yield kinetic luminosities ranging from $9.4 \times 10^{41} \mathrm{erg} \mathrm{s}^{-1}$ to $3.8 \times 10^{42} \mathrm{erg} \mathrm{s}^{-1}$. For the bolometric luminosity during our December 2016 observations of $2.8 \times 10^{44} \mathrm{erg} \mathrm{s}^{-1}$ (Mehdipour et al. 2017), and an Eddington luminosity of $2.8 \times 10^{45} \mathrm{erg} \mathrm{s}^{-1}$ (for $M_{\mathrm{BH}}=2.35 \times 10^{7} M_{\odot}$ ), the kinetic luminosity corresponds to a range of $0.34 \%-1.4 \%$ of bolometric, but only $0.03-0.14 \%$ of the Eddington luminosity. Even if this high-velocity outflow is persistent, these kinetic luminosities are not quite sufficient to have an evolutionary impact on the host galaxy, for which they generally must lie in the range of 0.5-5\% (Di Matteo et al. 2005; Hopkins \& Elvis 2010) of the AGN Eddington luminosity.

\section{Conclusions}

Our UV spectra of NGC 3783 obtained with HST/COS in simultaneity with $X M M-N e w t o n$ X-ray observations triggered with Swift by a soft X-ray obscuration event reveal that the obscurer is a fast, broad outflow similar in character to those recently discovered in NGC 5548 (Kaastra et al. 2014), Mrk 335 (Longinotti et al. 2013), and NGC 985 (Ebrero et al. 2016). The outflow extends from zero velocity to a maximum velocity of $-6200 \mathrm{~km} \mathrm{~s}^{-1}$, with a maximum depth at $-3000 \mathrm{~km} \mathrm{~s}^{-1}$, and a mean depth at $-2840 \mathrm{~km} \mathrm{~s}^{-1}$. The UV absorption is visible in Ly $\alpha, \mathrm{N} v$, Si Iv, and C Iv, indicating a moderate level of ionization. The X-ray determined column density of $N_{\mathrm{H}}=2.3 \times$ $10^{23} \mathrm{~cm}^{-2}$ together with the UV ion column densities yield an ionization parameter of $\log \xi=1.84_{-0.2}^{+0.4} \mathrm{erg} \mathrm{cm} \mathrm{s}^{-1}$, similar to highly ionized portions of the broad-line region.

Even though NGC 3783 was in a historically high UV flux state, the intrinsic absorption lines exhibit depths and ionization states similar to the lowest flux states seen in the past. The appearance of deep troughs and low-ionization states like C III demonstrate that despite the high UV continuum flux, the ionizing UV must be shadowed by the soft X-ray obscurer, similar to the behavior seen in NGC 5548 (Arav et al. 2015).

The broad emission line profiles in NGC 3783 changed dramatically as well. Despite the high UV continuum flux, the emission line fluxes are comparable to those seen with STIS in 2001, and moderate-width $\left(\sim 2800 \mathrm{~km} \mathrm{~s}^{-1}\right)$ portions of the broad-line profile have disappeared. We suggest that the central portions of the broad line region have collapsed in an infall, triggering the brightening observed in the UV and X-ray, and also triggering the obscuring outflow. Our observations illustrate the value of simultaneous UV and X-ray spectral observations to understand the kinematics and physical properties of obscuring events.

Acknowledgements. This work is based on observations obtained with the NASA/ESA HST, and obtained from the Hubble Legacy Archive. This work was supported by NASA through a grant for HST program number 14481 from the Space Telescope Science Institute, which is operated by the Association of Universities for Research in Astronomy, Incorporated, under NASA contract NAS5-26555. We also used XMM-Newton, an ESA science mission with instruments and contributions directly funded by ESA Member States and the USA (NASA), the NUSTAR mission, a project led by the California Institute of Technology (Caltech), managed by the Jet Propulsion Laboratory (JPL) and funded by NASA, and data supplied by the UK Swift Science Data Centre at the University of Leicester. We thank the XMM-Newton, NuSTAR, and HST teams for scheduling our Target of Opportunity triggered observations. SRON is supported financially by NWO, the Netherlands Organization for Scientific
Research. SB acknowledges financial support from the Italian Space Agency under grant ASI-INAF I/037/12/0, and n. 2017-14-H.O. EC is partially supported by the NWO-Vidi grant number 633.042.525. BDM acknowledges support from the European Union's Horizon 2020 research and innovation program under the Marie Skłodowska-Curie grant agreement No. 665778 via the Polish National Science Center grant Polonez UMO-2016/21/P/ST9/04025. POP acknowledges support from the CNES and CNRS/PNHE. GP acknowledges support from the Bundesministerium für Wirtschaft und Technologie/Deutsches Zentrum für Luftund Raumfahrt (BMWI/DLR, FKZ 50 OR 1604) and the Max Planck Society.

\section{References}

Anderson, J., \& Bedin, L. R. 2010, PASP, 122, 1035

Arav, N., Edmonds, D., Borguet, B., et al. 2012, A\&A, 544, A33

Arav, N., Chamberlain, C., Kriss, G. A., et al. 2015, A\&A, 577, A37

Baskin, A., \& Laor, A. 2018, MNRAS, 474, 1970

Bentz, M. C., \& Katz, S. 2015, PASP, 127, 67

Bentz, M. C., Walsh, J. L., Barth, A. J., et al. 2010, ApJ, 716, 993

Blandford, R. D., \& McKee, C. F. 1982, ApJ, 255, 419

Crenshaw, D. M., Kraemer, S. B., \& George, I. M. 2003, ARA\&A, 41, 117

Crenshaw, D. M., Kraemer, S. B., Schmitt, H. R., et al. 2009, ApJ, 698, 281

Czerny, B., \& Hryniewicz, K. 2011, A\&A, 525, L8

Czerny, B., Li, Y.-R., Hryniewicz, K., et al. 2017, ApJ, 846, 154

De Rosa, G., Peterson, B. M., Ely, J., et al. 2015, ApJ, 806, 128

Denney, K. D., De Rosa, G., Croxall, K., et al. 2014, ApJ, 796, 134

Di Matteo, T., Springel, V., Hernquist, L., et al. 2005, Nature, 433, 604

Dunn, J. P., Jackson, B., Deo, R. P., et al. 2006, PASP, 118, 572

Ebrero, J., Kriss, G. A., Kaastra, J. S., \& Ely, J. C. 2016, A\&A, 586, A72

Elitzur, M., \& Ho, L. C. 2009, ApJ, 701, L91

Elitzur, M., Ho, L. C., \& Trump, J. R. 2014, MNRAS, 438, 3340

Faucher-Giguère, C.-A., \& Quataert, E. 2012, MNRAS, 425, 605

Ferland, G. J., Chatzikos, M., Guzmán, F., et al. 2017, RMxAA, 53, 385

Ferrarese, L., \& Merritt, D. 2000, ApJ, 539, L9

Fukumura, K., Kazanas, D., Contopoulos, I., \& Behar, E. 2010, ApJ, 715, 636

Gabel, J. R., Crenshaw, D. M., Kraemer, S. B., et al. 2003a, ApJ, 583, 178 Gabel, J. R., Crenshaw, D. M., Kraemer, S. B., et al. 2003b, ApJ, 595, 120 Gabel, J. R., Kraemer, S. B., Crenshaw, D. M., et al. 2005, ApJ, 631, 741 Gebhardt, K., Bender, R., Bower, G., et al. 2000, ApJ, 539, L13

Gehrels, N., Chincarini, G., Giommi, P., et al. 2004, ApJ, 611, 1005 Goad, M. R., Korista, K. T., De Rosa, G., et al. 2016, ApJ, 824, 11 Green, J. C., Froning, C. S., Osterman, S., et al. 2012, ApJ, 744, 60 Grier, C. J., Peterson, B. M., Horne, K., et al. 2013, ApJ, 764, 47 Harrison, F. A., Craig, W. W., Christensen, F. E., et al. 2013, ApJ, 770, 103 Hopkins, P. F., \& Elvis, M. 2010, MNRAS, 401, 7

Horne, K., Peterson, B. M., Collier, S. J., \& Netzer, H. 2004, PASP, 116 , 465

Jansen, F., Lumb, D., Altieri, B., et al. 2001, A\&A, 365, L1

Kaastra, J. S., Kriss, G. A., Cappi, M., et al. 2014, Science, 345, 64 Kaastra, J. S., Mehdipour, M., Behar, E., et al. 2018, A\&A, 619, A112 Kaspi, S., Brandt, W. N., George, I. M., et al. 2002, ApJ, 574, 643 Kaufer, A., Stahl, O., Tubbesing, S., et al. 1999, The Messenger, 95, 8 Khachikian, E. Y., \& Weedman, D. W. 1971, ApJ, 164, L109

King, A. 2003, ApJ, 596, L27

Königl, A., \& Kartje, J. F. 1994, ApJ, 434, 446

Kormendy, J., \& Ho, L. C. 2013, ARA\&A, 51, 511

Kriss, G. 1994, Astron. Data Anal. Softw. Syst., 3, 437

Kriss, G. A., Arav, N., Kaastra, J. S., et al. 2011, A\&A, 534, A41

Krolik, J. H., \& Kriss, G. A. 1995, ApJ, 447, 512

Krolik, J. H., \& Kriss, G. A. 2001, ApJ, 561, 684

Krolik, J. H., Horne, K., Kallman, T. R., et al. 1991, ApJ, 371, 541

Liu, G., Zakamska, N. L., Greene, J. E., Nesvadba, N. P. H., \& Liu, X. 2013a, MNRAS, 430, 2327

Liu, G., Zakamska, N. L., Greene, J. E., Nesvadba, N. P. H., \& Liu, X. 2013b, MNRAS, 436, 2576

Liu, G., Zakamska, N. L., \& Greene, J. E. 2014, MNRAS, 442, 1303 Longinotti, A. L., Krongold, Y., Kriss, G. A., et al. 2013, ApJ, 766, 104 MacLeod, C. L., Green, P. J., Anderson, S. F., et al. 2018, ApJ, submitted, [arXiv:1810.00087]

MacLeod, C. L., Ross, N. P., Lawrence, A., et al. 2016, MNRAS, 457, 389

Magorrian, J., Tremaine, S., Richstone, D., et al. 1998, AJ, 115, 2285

Markowitz, A. G., Krumpe, M., \& Nikutta, R. 2014, MNRAS, 439, 1403

Mathur, S., Gupta, A., Page, K., et al. 2017, ApJ, 846, 55

Mathur, S., Denney, K. D., Gupta, A., et al. 2018, ApJ, 866, 123

Mehdipour, M., Kaastra, J. S., Kriss, G. A., et al. 2017, A\&A, 607, A28

Murphy, E. M., Lockman, F. J., Laor, A., \& Elvis, M. 1996, ApJS, 105, 369 
Murray, N., \& Chiang, J. 1995, ApJ, 454, L105

Murray, N., \& Chiang, J. 1997, ApJ, 474, 91

Nardini, E., Reeves, J. N., Gofford, J., et al. 2015, Science, 347, 860

Netzer, H., Kaspi, S., Behar, E., et al. 2003, ApJ, 599, 933

Onken, C. A., \& Peterson, B. M. 2002, ApJ, 572, 746

Osterbrock, D. E., \& Ferland, G. J. 2006, Astrophysics of Gaseous Nebulae and Active Galactic Nuclei (Mill Valley: University Science Books)

Ostriker, J. P., Choi, E., Ciotti, L., Novak, G. S., \& Proga, D. 2010, ApJ, 722, 642

Pancoast, A., Brewer, B. J., \& Treu, T. 2014a, MNRAS, 445, 3055

Pancoast, A., Brewer, B. J., Treu, T., et al. 2014b, MNRAS, 445, 3073

Penston, M. V., \& Perez, E. 1984, MNRAS, 211, 33P

Peterson, B. M. 1993, PASP, 105, 247

Peterson, B. M., \& Wandel, A. 1999, ApJ, 521, L95

Pounds, K. A., Reeves, J. N., King, A. R., et al. 2003, MNRAS, 345, 705

Proga, D., \& Waters, T. 2015, ApJ, 804, 137

Proga, D., Stone, J. M., \& Kallman, T. R. 2000, ApJ, 543, 686
Reeves, J. N., O’Brien, P. T., Braito, V., et al. 2009, ApJ, 701, 493 Reichert, G. A., Rodriguez-Pascual, P. M., Alloin, D., et al. 1994, ApJ, 425,582

Rumbaugh, N., Shen, Y., Morganson, E., et al. 2018, ApJ, 854, 160

Savage, B. D., \& Sembach, K. R. 1991, ApJ, 379, 245

Schlafly, E. F., \& Finkbeiner, D. P. 2011, ApJ, 737, 103

Scott, A. E., Brandt, W. N., Behar, E., et al. 2014, ApJ, 797, 105

Silk, J., \& Rees, M. J. 1998, A\&A, 331, L1

Soker, N. 2010, MNRAS, 407, 2355

Stirpe, G. M., Winge, C., Altieri, B., et al. 1994, ApJ, 425, 609

Theureau, G., Bottinelli, L., Coudreau-Durand, N., et al. 1998, A\&AS, 130, 333

Thompson, T. A., Fabian, A. C., Quataert, E., \& Murray, N. 2015, MNRAS, 449, 147

Tombesi, F., Cappi, M., Reeves, J. N., et al. 2010, A\&A, 521, A57

Woodgate, B. E., Kimble, R. A., Bowers, C. W., et al. 1998, PASP, 110, 1183

Zetzl, M., Kollatschny, W., Ochmann, M. W., et al. 2018, A\&A, 618, A83

Zubovas, K., \& Nayakshin, S. 2014, MNRAS, 440, 2625 


\section{Appendix A: Additional tables}

Table A.1. UV and optical observations of NGC 3783.

\begin{tabular}{|c|c|c|c|c|c|}
\hline Dataset name & Date & $\begin{array}{l}\text { Start time } \\
(\mathrm{GMT})\end{array}$ & $\begin{array}{l}\text { Start time } \\
(\mathrm{MJD})\end{array}$ & $\begin{array}{c}\text { Exposure time } \\
\text { (s) }\end{array}$ & Grating/tilt/FP-POS \\
\hline obgu03010 & $2011-03-23$ & $19: 41: 12$ & 55643.820286 & 696 & STIS/CCD/G430M \\
\hline obgu03020 & $2011-03-23$ & $19: 53: 58$ & 55643.829152 & 696 & STIS/CCD/G430M \\
\hline obgu03030 & 2011-03-23 & 21:11:46 & 55643.883179 & 696 & STIS/CCD/G430M \\
\hline lbgu19010 & $2011-05-26$ & $10: 40: 51$ & 55707.445041 & 481 & G130M/1291/3 \\
\hline lbgu19020 & $2011-05-26$ & $10: 52: 19$ & 55707.452998 & 481 & $\mathrm{G} 130 \mathrm{M} / 1300 / 3$ \\
\hline lbgu19030 & $2011-05-26$ & $11: 03: 47$ & 55707.460961 & 481 & G130M/1309/3 \\
\hline lbgu 19040 & $2011-05-26$ & $12: 05: 01$ & 55707.503484 & 481 & $\mathrm{G} 130 \mathrm{M} / 1318 / 3$ \\
\hline lbgu19050 & $2011-05-26$ & $12: 17: 11$ & 55707.511933 & 2425 & $\mathrm{G} 160 \mathrm{M} / 1589 / 3$ \\
\hline lbgu19060 & $2011-05-26$ & $12: 30: 34$ & 55707.521227 & 2425 & $\mathrm{G} 160 \mathrm{M} / 1600 / 3$ \\
\hline lbgu 19070 & $2011-05-26$ & $13: 46: 04$ & 55707.573657 & 2425 & $\mathrm{G} 160 \mathrm{M} / 1611 / 3$ \\
\hline lbgu19080 & $2011-05-26$ & $13: 59: 27$ & 55707.582951 & 2425 & $\mathrm{G} 160 \mathrm{M} / 1623 / 3$ \\
\hline $1 \mathrm{c} 3 \times 01010$ & 2013-03-30 & $14: 48: 30$ & 56381.617014 & 460 & G130M/1291/3 \\
\hline $1 \mathrm{c} 3 \mathrm{x} 01020$ & 2013-03-30 & $14: 59: 23$ & 56381.624572 & 460 & G130M/1300/3 \\
\hline lc $3 \times 01030$ & $2013-03-30$ & $15: 52: 17$ & 56381.661308 & 460 & G130M/1309/3 \\
\hline lc3x01040 & 2013-03-30 & $16: 03: 10$ & 56381.668866 & 340 & G130M/1318/3 \\
\hline lc $3 \times 01050$ & $2013-03-30$ & $16: 13: 24$ & 56381.675983 & 580 & G160M/1589/3 \\
\hline lc3x01060 & 2013-03-30 & $16: 26: 08$ & 56381.684815 & 580 & G160M/1600/3 \\
\hline $1 \mathrm{c} 3 \mathrm{x} 01070$ & $2013-03-30$ & $17: 28: 05$ & 56381.727836 & 580 & G160M/1611/3 \\
\hline $1 \mathrm{c} 3 \mathrm{x} 01080$ & 2013-03-30 & $17: 40: 49$ & 56381.736678 & 424 & G160M/1623/3 \\
\hline FEROS & 2016-12-12 & 07:36:00 & 57734.316667 & 1800 & FEROS \\
\hline $1 \mathrm{~d} 3 \mathrm{e} 03 \mathrm{kgq}^{a}$ & $2016-12-12$ & $12: 13: 56$ & 57734.509676 & 475 & G130M/1291/3 \\
\hline $1 \mathrm{~d} 3 \mathrm{e} 03 \mathrm{kkq}$ & 2016-12-12 & $12: 23: 55$ & 57734.516609 & 475 & G130M/1291/4 \\
\hline ld $3 \mathrm{e} 03 \mathrm{kmq}$ & $2016-12-12$ & $12: 35: 16$ & 57734.524502 & 455 & $\mathrm{G} 130 \mathrm{M} / 1327 / 1$ \\
\hline ld3e03koq & 2016-12-12 & $12: 44: 56$ & 57734.531204 & 455 & G130M/1327/2 \\
\hline ld3e03kyq & $2016-12-12$ & $13: 38: 36$ & 57734.568472 & 564 & $\mathrm{G} 160 \mathrm{M} / 1600 / 3$ \\
\hline $1 d 3 e 0312 q$ & 2016-12-12 & $13: 50: 04$ & 57734.576436 & 564 & G160M/1600/4 \\
\hline $1 d 3 e 0314 q$ & 2016-12-12 & $14: 02: 48$ & 57734.585278 & 564 & $\mathrm{G} 160 \mathrm{M} / 1623 / 1$ \\
\hline $1 d 3 e 0316 q$ & $2016-12-12$ & $14: 14: 16$ & 57734.593241 & 564 & $\mathrm{G} 160 \mathrm{M} / 1623 / 2$ \\
\hline $\operatorname{ld} 3 \mathrm{e} 04 \mathrm{pmq}^{a}$ & $2016-12-21$ & $15: 25: 00$ & 57743.642361 & 475 & $\mathrm{G} 130 \mathrm{M} / 1291 / 3$ \\
\hline ld $3 \mathrm{e} 04$ poq & 2016-12-21 & $15: 34: 59$ & 57743.649294 & 475 & G130M/1291/4 \\
\hline $1 \mathrm{~d} 3 \mathrm{e} 04 \mathrm{pqq}$ & $2016-12-21$ & $15: 46: 20$ & 57743.657187 & 455 & G130M/1327/1 \\
\hline ld3e04psq & $2016-12-21$ & $15: 56: 00$ & 57743.663889 & 455 & G130M/1327/2 \\
\hline ld3e04puq & $2016-12-21$ & $16: 07: 43$ & 57743.672026 & 564 & $\mathrm{G} 160 \mathrm{M} / 1600 / 3$ \\
\hline ld $3 \mathrm{e} 04 \mathrm{pwq}$ & 2016-12-21 & $17: 00: 22$ & 57743.708588 & 564 & $\mathrm{G} 160 \mathrm{M} / 1600 / 4$ \\
\hline ld3e04pyq & 2016-12-21 & 17:13:06 & 57743.717431 & 564 & $\mathrm{G} 160 \mathrm{M} / 1623 / 1$ \\
\hline $1 \mathrm{~d} 3 \mathrm{e} 04 \mathrm{q} 0 \mathrm{q}$ & $2016-12-21$ & $17: 24: 34$ & 57743.725394 & 564 & G160M/1623/2 \\
\hline
\end{tabular}

Notes. ${ }^{(a)}$ We collectively refer to the visits on 2016-12-12 as Visit 3, or "v3". ${ }^{(b)}$ We collectively refer to the visits on 2016-12-21 as Visit 4, or "v4". 
Table A.2. Emission-line parameters for the STIS 2000-2001 and COS 2016 spectra of NGC 3783.

\begin{tabular}{|c|c|c|c|c|c|c|c|}
\hline \multirow[b]{2}{*}{ Feature } & \multirow[b]{2}{*}{$\lambda_{0}{ }^{a}$} & \multicolumn{3}{|c|}{ STIS 2000-2001 } & \multicolumn{3}{|c|}{ COS 2016} \\
\hline & & Flux $^{b}$ & $v_{\text {sys }}{ }^{c}$ & $F W H M^{d}$ & Flux $^{b}$ & $v_{\text {sys }}{ }^{c}$ & $F W H M^{d}$ \\
\hline $\mathrm{C}_{\text {III }}$ & 1176.01 & $1.1 \pm 0.4$ & $-100 \pm 70$ & $1330 \pm 170$ & $40.0 \pm 4.4$ & $150 \pm 20$ & $1510 \pm 50$ \\
\hline $\operatorname{Ly} \alpha$ & 1215.67 & $84.0 \pm 2.7$ & $-40 \pm 20$ & $800 \pm 30$ & $100.0 \pm 3.8$ & $-40 \pm 20$ & $800 \pm 20$ \\
\hline $\operatorname{Ly} \alpha$ & 1215.67 & $240.0 \pm 9.6$ & $40 \pm 30$ & $2500 \pm 20$ & $270.0 \pm 8.1$ & $40 \pm 30$ & $2500 \pm 30$ \\
\hline $\operatorname{Ly} \alpha$ & 1215.67 & $120.0 \pm 4.6$ & $670 \pm 50$ & $5550 \pm 40$ & $290.0 \pm 9.7$ & $850 \pm 30$ & $6330 \pm 30$ \\
\hline $\operatorname{Ly} \alpha$ & 1215.67 & $300.0 \pm 9.8$ & $-270 \pm 30$ & $14940 \pm 100$ & $400.0 \pm 13.0$ & $150 \pm 40$ & $15960 \pm 60$ \\
\hline $\mathrm{N} v$ blue & 1238.82 & $6.4 \pm 0.2$ & $-40 \pm 20$ & $980 \pm 20$ & $7.6 \pm 0.3$ & $-40 \pm 30$ & $980 \pm 60$ \\
\hline $\mathrm{N} v$ red & 1242.80 & $6.4 \pm 0.2$ & $-40 \pm 20$ & $980 \pm 20$ & $7.6 \pm 0.3$ & $-40 \pm 30$ & $980 \pm 20$ \\
\hline $\mathrm{N} v$ blue & 1238.82 & $13.0 \pm 0.7$ & $350 \pm 40$ & $2850 \pm 40$ & $1.6 \pm 0.3$ & $350 \pm 70$ & $2850 \pm 120$ \\
\hline $\mathrm{N} v$ red & 1242.80 & $13.0 \pm 0.7$ & $350 \pm 40$ & $2850 \pm 40$ & $1.6 \pm 0.3$ & $350 \pm 70$ & $2850 \pm 120$ \\
\hline $\mathrm{Nv}$ & 1240.89 & $40.0 \pm 1.6$ & $660 \pm 60$ & $10380 \pm 180$ & $28.0 \pm 2.9$ & $1410 \pm 140$ & $12140 \pm 140$ \\
\hline $\mathrm{Si}$ II & 1260.42 & $1.7 \pm 0.1$ & $-150 \pm 20$ & $1600 \pm 20$ & $1.5 \pm 0.2$ & $-150 \pm 50$ & $1600 \pm 50$ \\
\hline $\mathrm{O}$ I+Si II & 1304.46 & $20.0 \pm 0.8$ & $0 \pm 30$ & $3460 \pm 40$ & $11.0 \pm 1.1$ & $220 \pm 50$ & $2500 \pm 40$ \\
\hline $\mathrm{C}_{\text {II }}$ & 1334.34 & $9.6 \pm 0.3$ & $0 \pm 50$ & $3460 \pm 40$ & $3.1 \pm 0.1$ & $110 \pm 40$ & $2500 \pm 20$ \\
\hline Si Iv blue & 1393.76 & $8.3 \pm 0.3$ & $-90 \pm 20$ & $1640 \pm 30$ & $4.3 \pm 0.3$ & $-280 \pm 50$ & $1170 \pm 70$ \\
\hline Si IV red & 1402.77 & $8.3 \pm 0.3$ & $-90 \pm 20$ & $1640 \pm 30$ & $4.3 \pm 0.3$ & $-280 \pm 50$ & $1170 \pm 20$ \\
\hline Si iv blue & 1393.76 & $23.0 \pm 0.7$ & $120 \pm 20$ & $4460 \pm 30$ & $23.0 \pm 0.9$ & $-270 \pm 30$ & $5160 \pm 60$ \\
\hline Si IV red & 1402.77 & $23.0 \pm 0.7$ & $120 \pm 20$ & $4460 \pm 30$ & $23.0 \pm 0.9$ & $-270 \pm 30$ & $5160 \pm 20$ \\
\hline Si IV & 1398.19 & $63.0 \pm 1.9$ & $-1170 \pm 30$ & $12410 \pm 80$ & $55.0 \pm 2.3$ & $-2730 \pm 40$ & $13480 \pm 290$ \\
\hline $\left.\mathrm{O}_{\mathrm{IV}}\right]$ & 1400.37 & $5.2 \pm 0.4$ & $0 \pm 20$ & $1640 \pm 80$ & $0.8 \pm 0.1$ & $900 \pm 60$ & $1170 \pm 20$ \\
\hline O IV] & 1400.37 & & $\ldots$ & & $9.1 \pm 0.5$ & $900 \pm 60$ & $5160 \pm 20$ \\
\hline $\mathrm{N}$ IV] & 1485.80 & $14.0 \pm 0.5$ & $-10 \pm 40$ & $2600 \pm 40$ & $2.9 \pm 0.4$ & $-130 \pm 80$ & $1170 \pm 20$ \\
\hline N IV] & $14 z 5.80$ & & & & $8.3 \pm 0.4$ & $-130 \pm 80$ & $3250 \pm 20$ \\
\hline C iv blue & 1548.19 & $40.0 \pm 1.7$ & $-60 \pm 20$ & $940 \pm 40$ & $36.0 \pm 1.7$ & $-60 \pm 30$ & $940 \pm 30$ \\
\hline C IV red & 1550.77 & $40.0 \pm 1.7$ & $-60 \pm 20$ & $940 \pm 40$ & $36.0 \pm 1.7$ & $-60 \pm 30$ & $940 \pm 20$ \\
\hline C Iv blue & 1548.19 & $80.0 \pm 2.6$ & $140 \pm 20$ & $2840 \pm 20$ & $2.3 \pm 0.6$ & $140 \pm 20$ & $2840 \pm 20$ \\
\hline $\mathrm{C}_{\text {IV }}$ red & 1550.77 & $80.0 \pm 2.6$ & $140 \pm 20$ & $2840 \pm 20$ & $2.3 \pm 0.6$ & $140 \pm 20$ & $2840 \pm 20$ \\
\hline C Iv blue & 1548.19 & $84.0 \pm 2.7$ & $-950 \pm 20$ & $4580 \pm 30$ & $180.0 \pm 5.5$ & $-710 \pm 30$ & $4640 \pm 30$ \\
\hline C IV red & 1550.77 & $84.0 \pm 2.7$ & $-950 \pm 20$ & $4580 \pm 30$ & $180.0 \pm 5.5$ & $-710 \pm 30$ & $4640 \pm 30$ \\
\hline C IV & 1549.48 & $310.0 \pm 9.3$ & $-60 \pm 20$ & $10030 \pm 30$ & $340.0 \pm 11.0$ & $-70 \pm 30$ & $12710 \pm 40$ \\
\hline He II & 1640.45 & $13.0 \pm 0.5$ & $-140 \pm 20$ & $990 \pm 20$ & $14.0 \pm 0.6$ & $10 \pm 50$ & $820 \pm 30$ \\
\hline He II & 1640.45 & $9.2 \pm 0.3$ & $-510 \pm 40$ & $3000 \pm 100$ & $7.6 \pm 1.2$ & $1250 \pm 40$ & $3990 \pm 900$ \\
\hline He II & 1640.45 & $140.0 \pm 4.4$ & $-470 \pm 30$ & $11280 \pm 30$ & $160.0 \pm 6.3$ & $-120 \pm 30$ & $12460 \pm 50$ \\
\hline O III] & 1659.85 & $3.6 \pm 0.2$ & $0 \pm 50$ & $1200 \pm 100$ & $3.1 \pm 0.4$ & $150 \pm 40$ & $1210 \pm 30$ \\
\hline O III] & 1665.19 & $7.6 \pm 0.6$ & $0 \pm 10$ & $1200 \pm 100$ & $5.7 \pm 0.4$ & $150 \pm 20$ & $1210 \pm 20$ \\
\hline $\mathrm{N}$ III] & 1750.00 & $\ldots$ & $\ldots$ & $\ldots$ & $21.0 \pm 0.7$ & $0 \pm 30$ & $3270 \pm 40$ \\
\hline
\end{tabular}

Notes. ${ }^{(a)}$ Vacuum rest wavelength of the spectral feature $(\AA) .{ }^{(b)}$ Integrated flux in units of $10^{-14} \mathrm{erg} \mathrm{cm}^{-2} \mathrm{~s}^{-1}$. ${ }^{(c)}$ Velocity (in km s$\left.{ }^{-1}\right)$ relative to a systemic redshift of $z=0.00973$ (Theureau et al. 1998). ${ }^{(d)}$ Full-width at half-maximum $\left(\mathrm{km} \mathrm{s}^{-1}\right)$. 
G. A. Kriss et al.: HST/COS observations of the obscuring outflow in NGC 3783

Table A.3. Emission-line parameters for the individual COS 2016 spectra of NGC 3783.

\begin{tabular}{|c|c|c|c|c|c|c|c|}
\hline \multirow[b]{2}{*}{ Feature } & \multirow[b]{2}{*}{$\lambda_{0}{ }^{a}$} & \multicolumn{3}{|c|}{ COS 2016-12-12 } & \multicolumn{3}{|c|}{ COS 2016-12-21 } \\
\hline & & Flux $^{b}$ & $v_{\mathrm{sys}}{ }^{c}$ & $F W H M^{d}$ & Flux $^{b}$ & $v_{\mathrm{sys}}{ }^{c}$ & $F W H M^{d}$ \\
\hline $\mathrm{C}_{\text {III }}$ & 1176.01 & $47.0 \pm 2.6$ & $170 \pm 110$ & $1490 \pm 50$ & $34.0 \pm 1.5$ & $130 \pm 20$ & $1520 \pm 60$ \\
\hline $\operatorname{Ly} \alpha$ & 1215.67 & $120.0 \pm 4.4$ & $-40 \pm 30$ & $800 \pm 30$ & $120.0 \pm 4.0$ & $-40 \pm 20$ & $800 \pm 20$ \\
\hline $\operatorname{Ly} \alpha$ & 1215.67 & $250.0 \pm 8.2$ & $-240 \pm 40$ & $4190 \pm 40$ & $340.0 \pm 12.0$ & $-240 \pm 20$ & $4190 \pm 40$ \\
\hline $\operatorname{Ly} \alpha$ & 1215.67 & $350.0 \pm 11.0$ & $-70 \pm 30$ & $6950 \pm 20$ & $310.0 \pm 13.0$ & $-70 \pm 30$ & $6950 \pm 70$ \\
\hline $\operatorname{Ly} \alpha$ & 1215.67 & $320.0 \pm 9.8$ & $60 \pm 40$ & $18010 \pm 70$ & $320.0 \pm 11.0$ & $60 \pm 20$ & $18010 \pm 30$ \\
\hline N v blue & 1238.82 & $5.1 \pm 0.2$ & $-40 \pm 30$ & $980 \pm 30$ & $7.8 \pm 1.2$ & $-40 \pm 30$ & $980 \pm 50$ \\
\hline $\mathrm{N} v$ red & 1242.80 & $5.1 \pm 0.2$ & $-40 \pm 30$ & $980 \pm 30$ & $7.8 \pm 1.2$ & $-40 \pm 30$ & $980 \pm 50$ \\
\hline Nv blue & 1238.82 & $7.4 \pm 0.3$ & $-30 \pm 30$ & $2850 \pm 40$ & $9.9 \pm 0.6$ & $-30 \pm 60$ & $2850 \pm 20$ \\
\hline $\mathrm{N} v$ red & 1242.80 & $7.4 \pm 0.3$ & $-30 \pm 30$ & $2850 \pm 40$ & $9.9 \pm 0.6$ & $-30 \pm 60$ & $2850 \pm 20$ \\
\hline $\mathrm{Nv}$ & 1240.89 & $25.0 \pm 1.0$ & $-220 \pm 170$ & $12530 \pm 530$ & $73.0 \pm 2.6$ & $-220 \pm 50$ & $12530 \pm 40$ \\
\hline Si II & 1260.42 & $1.5 \pm 0.1$ & $-150 \pm 30$ & $1600 \pm 40$ & $1.7 \pm 0.2$ & $-150 \pm 50$ & $1600 \pm 120$ \\
\hline $\mathrm{O}_{\mathrm{I}}+\mathrm{Si}$ II & 1304.46 & $10.0 \pm 1.0$ & $220 \pm 30$ & $2500 \pm 50$ & $11.0 \pm 0.4$ & $220 \pm 40$ & $2500 \pm 60$ \\
\hline $\mathrm{C}_{\text {II }}$ & 1334.53 & $2.7 \pm 0.2$ & $110 \pm 40$ & $2500 \pm 20$ & $2.8 \pm 0.2$ & $110 \pm 50$ & $2500 \pm 20$ \\
\hline Si Iv blue & 1393.76 & $4.6 \pm 0.2$ & $-170 \pm 50$ & $1110 \pm 40$ & $4.6 \pm 0.2$ & $-170 \pm 30$ & $1110 \pm 70$ \\
\hline Si IV red & 1402.77 & $4.6 \pm 0.2$ & $-170 \pm 50$ & $1110 \pm 20$ & $4.6 \pm 0.2$ & $-170 \pm 30$ & $1110 \pm 70$ \\
\hline Si Iv blue & 1393.76 & $17.0 \pm 0.8$ & $-270 \pm 20$ & $5140 \pm 60$ & $28.0 \pm 1.2$ & $-270 \pm 30$ & $5140 \pm 80$ \\
\hline Si IV red & 1402.77 & $17.0 \pm 0.8$ & $-270 \pm 20$ & $5140 \pm 60$ & $28.0 \pm 1.2$ & $-270 \pm 30$ & $5140 \pm 80$ \\
\hline Si IV & 1398.19 & $62.0 \pm 2.3$ & $-2750 \pm 30$ & $13030 \pm 80$ & $51.0 \pm 1.7$ & $-2750 \pm 40$ & $13030 \pm 140$ \\
\hline O IV] & 1401.16 & $0.7 \pm 0.1$ & $900 \pm 30$ & $1110 \pm 20$ & $0.7 \pm 0.1$ & $900 \pm 40$ & $1110 \pm 20$ \\
\hline O IV] & 1401.16 & $9.1 \pm 1.0$ & $900 \pm 30$ & $5140 \pm 20$ & $9.1 \pm 0.4$ & $900 \pm 40$ & $5140 \pm 20$ \\
\hline $\left.\mathrm{N}_{\text {IV }}\right]$ & 1486.50 & $4.1 \pm 0.4$ & $-140 \pm 20$ & $1110 \pm 20$ & $2.5 \pm 0.3$ & $-80 \pm 20$ & $1110 \pm 20$ \\
\hline $\mathrm{N}$ IV] & 1486.50 & $11.0 \pm 0.8$ & $-140 \pm 20$ & $4330 \pm 60$ & $6.3 \pm 0.9$ & $-80 \pm 20$ & $2690 \pm 20$ \\
\hline C Iv blue & 1548.19 & $35.0 \pm 1.1$ & $-60 \pm 20$ & $940 \pm 20$ & $38.0 \pm 1.9$ & $-60 \pm 20$ & $940 \pm 30$ \\
\hline C IV red & 1550.77 & $35.0 \pm 1.1$ & $-60 \pm 20$ & $940 \pm 20$ & $38.0 \pm 1.9$ & $-60 \pm 20$ & $940 \pm 30$ \\
\hline Civ blue & 1548.19 & $3.3 \pm 0.2$ & $140 \pm 50$ & $2840 \pm 20$ & $4.8 \pm 0.3$ & $140 \pm 20$ & $2840 \pm 70$ \\
\hline C IV red & 1550.77 & $3.3 \pm 0.2$ & $140 \pm 50$ & $2840 \pm 20$ & $4.8 \pm 0.3$ & $140 \pm 20$ & $2840 \pm 70$ \\
\hline $\mathrm{C}$ Iv blue & 1548.19 & $190.0 \pm 5.7$ & $-790 \pm 20$ & $4800 \pm 20$ & $160.0 \pm 5.0$ & $-570 \pm 30$ & $4530 \pm 30$ \\
\hline $\mathrm{C}_{\text {IV red }}$ & 1550.77 & $190.0 \pm 5.7$ & $-790 \pm 20$ & $4800 \pm 20$ & $160.0 \pm 5.0$ & $-570 \pm 30$ & $4530 \pm 30$ \\
\hline $\mathrm{C}_{\text {IV }}$ & 1549.48 & $310.0 \pm 11.0$ & $-220 \pm 30$ & $13000 \pm 120$ & $370.0 \pm 13.0$ & $120 \pm 30$ & $12500 \pm 50$ \\
\hline $\mathrm{He}_{\text {II }}$ & 1640.45 & $14.0 \pm 0.7$ & $40 \pm 20$ & $910 \pm 30$ & $14.0 \pm 1.4$ & $0 \pm 30$ & $790 \pm 30$ \\
\hline He II & 1640.45 & $7.9 \pm 0.3$ & $2100 \pm 100$ & $5090 \pm 120$ & $8.7 \pm 0.4$ & $1400 \pm 110$ & $3490 \pm 270$ \\
\hline He II & 1640.45 & $150.0 \pm 4.8$ & $-120 \pm 30$ & $11860 \pm 60$ & $170.0 \pm 5.2$ & $-120 \pm 20$ & $12530 \pm 100$ \\
\hline $\mathrm{O}$ III] & 1660.81 & $2.3 \pm 0.3$ & $160 \pm 50$ & $1220 \pm 60$ & $3.2 \pm 0.1$ & $160 \pm 30$ & $1210 \pm 30$ \\
\hline $\mathrm{O}$ III] & 1666.15 & $4.3 \pm 0.5$ & $150 \pm 20$ & $1220 \pm 20$ & $6.8 \pm 0.4$ & $160 \pm 20$ & $1210 \pm 20$ \\
\hline $\mathrm{N}_{\text {III] }}$ & 1750.00 & $21.0 \pm 0.8$ & $0 \pm 20$ & $3270 \pm 30$ & $21.0 \pm 0.6$ & $0 \pm 20$ & $3270 \pm 20$ \\
\hline
\end{tabular}

Notes. ${ }^{(a)}$ Vacuum rest wavelength of the spectral feature $(\AA)$. ${ }^{(b)}$ Integrated flux in units of $10^{-14} \mathrm{erg} \mathrm{cm}^{-2} \mathrm{~s}^{-1}$. ${ }^{(c)}$ Velocity (in km s$\left.{ }^{-1}\right)$ relative to a systemic redshift of $z=0.00973$ (Theureau et al. 1998). ${ }^{(d)}$ Full-width at half-maximum $\left(\mathrm{km} \mathrm{s}^{-1}\right)$. 
Table A.4. Emission-line parameters for the COS 2011 and COS 2013 spectra of NGC 3783.

\begin{tabular}{|c|c|c|c|c|c|c|c|}
\hline \multirow[b]{2}{*}{ Feature } & \multirow[b]{2}{*}{$\lambda_{0}{ }^{a}$} & \multicolumn{3}{|c|}{ COS 2011} & \multicolumn{3}{|c|}{ COS 2013} \\
\hline & & Flux $^{b}$ & $v_{\mathrm{sys}}{ }^{c}$ & $F W H M^{d}$ & Flux $^{b}$ & $\nu_{\mathrm{sys}}^{c}$ & $F W H M^{d}$ \\
\hline $\mathrm{C}_{\text {III }}$ & 1176.01 & $1.1 \pm 0.2$ & $-100 \pm 80$ & $1200 \pm 180$ & $1.5 \pm 0.3$ & $0 \pm 50$ & $1200 \pm 260$ \\
\hline $\operatorname{Ly} \alpha$ & 1215.67 & $110.0 \pm 3.7$ & $-110 \pm 20$ & $800 \pm 20$ & $100.0 \pm 3.1$ & $-40 \pm 10$ & $800 \pm 20$ \\
\hline $\operatorname{Ly} \alpha$ & 1215.67 & $150.0 \pm 5.9$ & $180 \pm 20$ & $2430 \pm 30$ & $170.0 \pm 5.8$ & $90 \pm 10$ & $2310 \pm 10$ \\
\hline $\operatorname{Ly} \alpha$ & 1215.67 & $260.0 \pm 9.3$ & $890 \pm 60$ & $5190 \pm 60$ & $77.0 \pm 3.9$ & $1760 \pm 40$ & $6070 \pm 50$ \\
\hline $\operatorname{Ly} \alpha$ & 1215.67 & $230.0 \pm 7.6$ & $-1160 \pm 60$ & $14870 \pm 50$ & $160.0 \pm 5.3$ & $200 \pm 20$ & $10590 \pm 30$ \\
\hline Nv blue & 1238.82 & $5.1 \pm 0.3$ & $-30 \pm 30$ & $770 \pm 80$ & $7.4 \pm 0.3$ & $-30 \pm 10$ & $770 \pm 20$ \\
\hline $\mathrm{N} v$ red & 1242.80 & $5.1 \pm 0.3$ & $-30 \pm 30$ & $770 \pm 80$ & $7.4 \pm 0.3$ & $-30 \pm 10$ & $770 \pm 20$ \\
\hline $\mathrm{N} v$ blue & 1238.82 & $6.0 \pm 0.4$ & $1080 \pm 20$ & $4240 \pm 60$ & $11.0 \pm 0.5$ & $580 \pm 20$ & $3690 \pm 20$ \\
\hline Nv red & 1242.80 & $6.0 \pm 0.4$ & $1080 \pm 20$ & $4240 \pm 60$ & $11.0 \pm 0.5$ & $580 \pm 20$ & $3690 \pm 20$ \\
\hline $\mathrm{Nv}$ & 1240.89 & $89.0 \pm 3.0$ & $480 \pm 20$ & $7270 \pm 60$ & $2.8 \pm 0.4$ & $450 \pm 930$ & $13100 \pm 720$ \\
\hline $\mathrm{S}_{\text {II }}$ & 1260.42 & $1.1 \pm 0.5$ & $-150 \pm 20$ & $1600 \pm 40$ & $6.4 \pm 0.4$ & $-150 \pm 30$ & $2460 \pm 30$ \\
\hline $\mathrm{O}_{\mathrm{I}}+\mathrm{S}_{\mathrm{II}}$ & 1304.46 & $11.0 \pm 0.8$ & $220 \pm 20$ & $2880 \pm 100$ & $11.0 \pm 0.4$ & $220 \pm 20$ & $2920 \pm 30$ \\
\hline $\mathrm{C}_{\text {II }}$ & 1334.53 & $4.3 \pm 0.4$ & $960 \pm 50$ & $2880 \pm 100$ & $4.0 \pm 0.2$ & $110 \pm 30$ & $2920 \pm 30$ \\
\hline S IV blue & 1393.76 & $4.3 \pm 0.4$ & $-280 \pm 30$ & $1310 \pm 100$ & $3.6 \pm 0.2$ & $-70 \pm 50$ & $1260 \pm 30$ \\
\hline S IV red & 1402.77 & $4.3 \pm 0.4$ & $-510 \pm 90$ & $1310 \pm 20$ & $3.6 \pm 0.2$ & $-70 \pm 50$ & $1260 \pm 30$ \\
\hline S Iv blue & 1393.76 & $19.0 \pm 0.6$ & $1060 \pm 20$ & $4740 \pm 50$ & $23.0 \pm 1.3$ & $-120 \pm 10$ & $5920 \pm 100$ \\
\hline S IV red & 1402.77 & $19.0 \pm 0.6$ & $1060 \pm 20$ & $4740 \pm 50$ & $23.0 \pm 1.3$ & $-120 \pm 10$ & $5920 \pm 100$ \\
\hline S IV & 1398.19 & $42.0 \pm 1.4$ & $-360 \pm 50$ & $10850 \pm 50$ & $15.0 \pm 1.2$ & $-3060 \pm 70$ & $17800 \pm 50$ \\
\hline O IV] & 1401.16 & $1.8 \pm 0.1$ & $700 \pm 40$ & $1400 \pm 130$ & $2.1 \pm 0.3$ & $900 \pm 40$ & $1260 \pm 10$ \\
\hline O IV] & 1401.16 & $11.0 \pm 0.6$ & $700 \pm 40$ & $4740 \pm 20$ & $1.9 \pm 0.4$ & $900 \pm 40$ & $5920 \pm 10$ \\
\hline $\mathrm{N}$ IV] & & & $10 \pm 30$ & $5000 \pm 190$ & $0.7 \pm 0.4$ & $10 \pm 20$ & $500 \pm 20$ \\
\hline $\mathrm{N}$ IV] & 1486.50 & $12.0 \pm 0.5$ & $-260 \pm 20$ & $2590 \pm 60$ & $9.5 \pm 0.4$ & $-260 \pm 10$ & $2590 \pm 70$ \\
\hline C Iv blue & 1548.19 & $40.0 \pm 1.5$ & $-60 \pm 20$ & $940 \pm 20$ & $37.0 \pm 1.6$ & $-60 \pm 20$ & $940 \pm 10$ \\
\hline Civ red & 1550.77 & $40.0 \pm 1.5$ & $-60 \pm 20$ & $940 \pm 20$ & $37.0 \pm 1.6$ & $-60 \pm 20$ & $940 \pm 10$ \\
\hline Civ blue & 1548.19 & $7.9 \pm 0.8$ & $300 \pm 80$ & $2840 \pm 50$ & $22.0 \pm 1.6$ & $500 \pm 30$ & $1390 \pm 70$ \\
\hline $\mathrm{C}_{\text {IV }}$ red & 1550.77 & $7.9 \pm 0.8$ & $300 \pm 80$ & $2840 \pm 50$ & $22.0 \pm 1.6$ & $500 \pm 30$ & $1390 \pm 70$ \\
\hline $\mathrm{C}_{\text {IV }}$ blue & 1548.19 & $140.0 \pm 4.7$ & $500 \pm 20$ & $4580 \pm 20$ & $68.0 \pm 2.3$ & $-620 \pm 30$ & $5030 \pm 40$ \\
\hline $\mathrm{C}_{\text {IV }}$ red & 1550.77 & $140.0 \pm 4.7$ & $500 \pm 20$ & $4580 \pm 20$ & $68.0 \pm 2.3$ & $-620 \pm 30$ & $5030 \pm 40$ \\
\hline $\mathrm{C}_{\mathrm{IV}}$ & 1549.48 & $300.0 \pm 10.0$ & $-430 \pm 20$ & $9840 \pm 30$ & $250.0 \pm 7.9$ & $-140 \pm 10$ & $9220 \pm 20$ \\
\hline He II & 1640.45 & $9.1 \pm 0.6$ & $-80 \pm 40$ & $790 \pm 50$ & $6.9 \pm 0.3$ & $90 \pm 20$ & $790 \pm 40$ \\
\hline He II & 1640.45 & $15.0 \pm 0.6$ & $840 \pm 90$ & $3250 \pm 30$ & $19.0 \pm 1.1$ & $-110 \pm 30$ & $3450 \pm 120$ \\
\hline He II & 1640.45 & $98.0 \pm 3.0$ & $-120 \pm 20$ & $11080 \pm 30$ & $39.0 \pm 1.9$ & $-5330 \pm 40$ & $23200 \pm 660$ \\
\hline $\left.\mathrm{O}_{\mathrm{III}}\right]$ & 1660.81 & $5.5 \pm 0.3$ & $-160 \pm 20$ & $1200 \pm 20$ & $3.5 \pm 0.4$ & $-160 \pm 20$ & $2790 \pm 60$ \\
\hline O III] & 1666.15 & $6.5 \pm 0.3$ & $-160 \pm 20$ & $1200 \pm 20$ & $15.0 \pm 0.7$ & $-160 \pm 10$ & $2790 \pm 60$ \\
\hline $\left.\mathrm{N}_{\text {III }}\right]$ & 1750.00 & $13.0 \pm 0.4$ & $0 \pm 30$ & $3270 \pm 20$ & $15.0 \pm 0.5$ & $0 \pm 40$ & $3270 \pm 30$ \\
\hline
\end{tabular}

Notes. ${ }^{(a)}$ Vacuum rest wavelength of the spectral feature $(\AA) .{ }^{(b)}$ Integrated flux in units of $10^{-14} \mathrm{erg} \mathrm{cm}^{-2} \mathrm{~s}^{-1}$. ${ }^{(c)}$ Velocity (in km s$\left.{ }^{-1}\right)$ relative to a systemic redshift of $z=0.00973$ (Theureau et al. 1998). ${ }^{(d)}$ Full-width at half-maximum $\left(\mathrm{km} \mathrm{s}^{-1}\right)$. 